\title{
Convexity, Classification, and Risk Bounds
}

\author{
Peter L. Bartlett \\ Division of Computer Science and Department of Statistics \\ University of California, Berkeley \\ bartlett@stat.berkeley.edu \\ Michael I. Jordan \\ Division of Computer Science and Department of Statistics \\ University of California, Berkeley \\ jordan@stat. berkeley. edu \\ Jon D. McAuliffe \\ Department of Statistics \\ University of California, Berkeley \\ jon@stat . berkeley.edu
}

November 4, 2003

Technical Report 638

\begin{abstract}
Many of the classification algorithms developed in the machine learning literature, including the support vector machine and boosting, can be viewed as minimum contrast methods that minimize a convex surrogate of the 0-1 loss function. The convexity makes these algorithms computationally efficient. The use of a surrogate, however, has statistical consequences that must be balanced against the computational virtues of convexity. To study these issues, we provide a general quantitative relationship between the risk as assessed using the 0-1 loss and the risk as assessed using any nonnegative surrogate loss function. We show that this relationship gives nontrivial upper bounds on excess risk under the weakest possible condition on the loss function: that it satisfy a pointwise form of Fisher consistency for classification. The relationship is based on a simple variational transformation of the loss function that is easy to compute in many applications. We also present a refined version of this result in the case of low noise. Finally, we present applications of our results to the estimation of convergence rates in the general setting of function classes that are scaled convex hulls of a finite-dimensional base class, with a variety of commonly used loss functions.
\end{abstract}

Keywords: machine learning, convex optimization, boosting, support vector machine, Rademacher complexity, empirical process theory 


\section{Introduction}

Convexity has become an increasingly important theme in applied mathematics and engineering, having acquired a prominent role akin to the one played by linearity for many decades. Building on the discovery of efficient algorithms for linear programs, researchers in convex optimization theory have developed computationally tractable methods for large classes of convex programs (Nesterov and Nemirovskii, 1994). Many fields in which optimality principles form the core conceptual structure have been changed significantly by the introduction of these new techniques (Boyd and Vandenberghe, 2003).

Convexity arises in many guises in statistics as well, notably in properties associated with the exponential family of distributions (Brown, 1986). It is, however, only in recent years that the systematic exploitation of the algorithmic consequences of convexity has begun in statistics. One applied area in which this trend has been most salient is machine learning, where the focus has been on large-scale statistical models for which computational efficiency is an imperative. Many of the most prominent methods studied in machine learning make significant use of convexity; in particular, support vector machines (Boser et al., 1992, Cortes and Vapnik, 1995, Cristianini and Shawe-Taylor, 2000, Schölkopf and Smola, 2002), boosting (Freund and Schapire, 1997, Collins et al., 2002, Lebanon and Lafferty, 2002), and variational inference for graphical models (Jordan et al., 1999) are all based directly on ideas from convex optimization.

If algorithms from convex optimization are to continue to make inroads into statistical theory and practice, it is important that we understand these algorithms not only from a computational point of view but also in terms of their statistical properties. What are the statistical consequences of choosing models and estimation procedures so as to exploit the computational advantages of convexity?

In the current paper we study this question in the context of multivariate classification. We consider the setting in which a covariate vector $X \in \mathcal{X}$ is to be classified according to a binary response $Y \in\{-1,1\}$. The goal is to choose a discriminant function $f: \mathcal{X} \rightarrow \mathbb{R}$, from a class of functions $\mathcal{F}$, such that the sign of $f(X)$ is an accurate prediction of $Y$ under an unknown joint measure $P$ on $(X, Y)$. We focus on $0-1$ loss; thus, letting $\ell(\alpha)$ denote an indicator function that is one if $\alpha \leq 0$ and zero otherwise, we wish to choose $f \in \mathcal{F}$ that minimizes the risk $R(f)=$ $\mathbf{E} \ell(Y f(X))=P(Y \neq \operatorname{sign}(f(X)))$.

Given a sample $D_{n}=\left(\left(X_{1}, Y_{1}\right), \ldots,\left(X_{n}, Y_{n}\right)\right)$, it is natural to consider estimation procedures based on minimizing the sample average of the loss, $\hat{R}(f)=\frac{1}{n} \sum_{i=1}^{n} \ell\left(Y_{i} f\left(X_{i}\right)\right)$. As is well known, however, such a procedure is computationally intractable for many nontrivial classes of functions (see, e.g., Arora et al., 1997). Indeed, the loss function $\ell(Y f(X))$ is non-convex in its (scalar) argument, and, while not a proof, this suggests a source of the difficulty. Moreover, it suggests that we might base a tractable estimation procedure on minimization of a convex surrogate $\phi(\alpha)$ for the loss. In particular, if $\mathcal{F}$ consists of functions that are linear in a parameter vector $\theta$, then the overall problem of minimizing expectations of $\phi(Y f(X))$ is convex in $\theta$. Given a convex parameter space, we obtain a convex program and can exploit the methods of convex optimization. A wide variety of classification methods in machine learning are based on this tactic; in particular, Figure 1 shows the (upper-bounding) convex surrogates associated with the support vector machine (Cortes and Vapnik, 1995), Adaboost (Freund and Schapire, 1997) and logistic regression (Friedman et al., 2000).

A basic statistical understanding of this setting has begun to emerge. In particular, when 


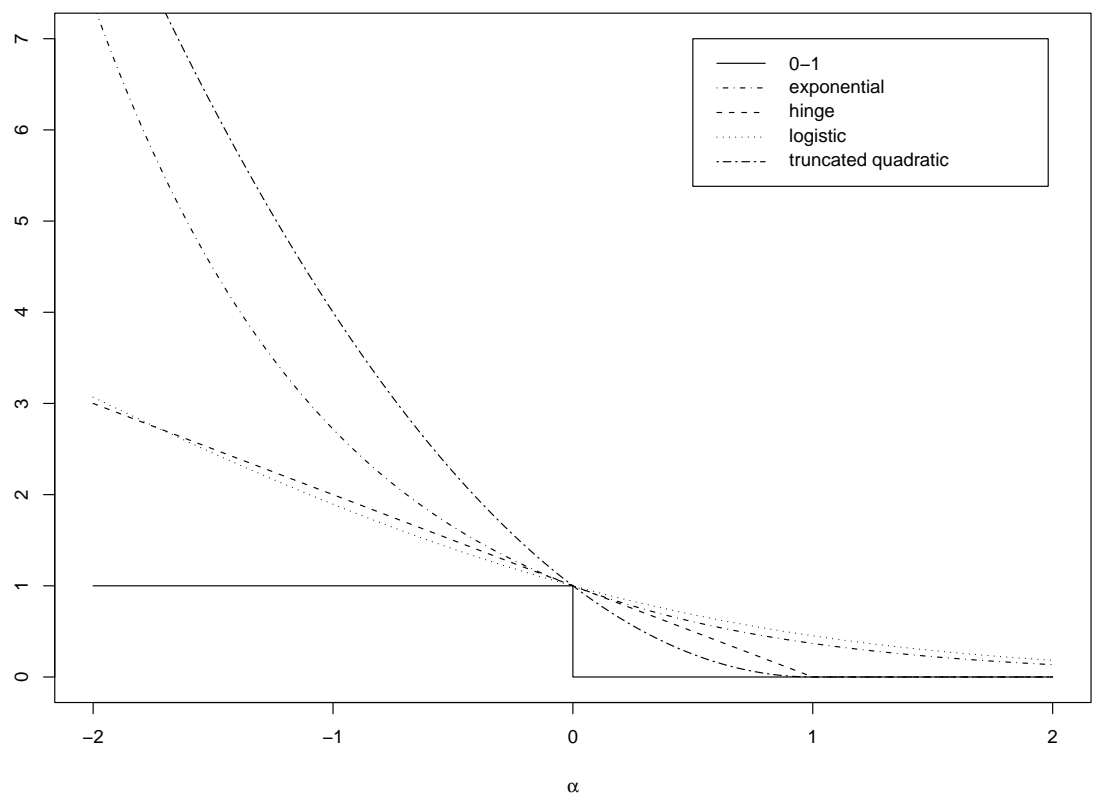

Figure 1: A plot of the 0-1 loss function and surrogates corresponding to various practical classifiers. These functions are plotted as a function of the margin $\alpha=y f(x)$. Note that a classification error is made if and only if the margin is negative; thus the 0-1 loss is a step function that is equal to one for negative values of the abscissa. The curve labeled "logistic" is the negative log likelihood, or deviance, under a logistic regression model; "hinge" is the piecewise-linear loss used in the support vector machine; and "exponential" is the exponential loss used by the Adaboost algorithm. The deviance is scaled so as to majorize the 0-1 loss; see Lemma 9.

appropriate regularization conditions are imposed, it is possible to demonstrate the Bayes-risk consistency of methods based on minimizing convex surrogates for 0-1 loss. Lugosi and Vayatis (2003) have provided such a result under the assumption that the surrogate $\phi$ is differentiable, monotone, strictly convex, and satisfies $\phi(0)=1$. This handles all of the cases shown in Figure 1 except the support vector machine. Steinwart (2002) has demonstrated consistency for the support vector machine as well, in a general setting where $\mathcal{F}$ is taken to be a reproducing kernel Hilbert space, and $\phi$ is assumed continuous. Other results on Bayes-risk consistency have been presented by Breiman (2000), Jiang (2003), Mannor and Meir (2001), and Mannor et al. (2002).

Consistency results provide reassurance that optimizing a surrogate does not ultimately hinder the search for a function that achieves the Bayes risk, and thus allow such a search to proceed within the scope of computationally efficient algorithms. There is, however, an additional motivation for working with surrogates of $0-1$ loss beyond the computational imperative. Minimizing the sample average of an appropriately-behaved loss function has a regularizing effect: it is possible to obtain uniform upper bounds on the risk of a function that minimizes the empirical average of the loss $\phi$, even for classes that are so rich that no such upper bounds are possible for the minimizer of the empirical average of the 0-1 loss. Indeed a number of such results have been obtained for function classes with infinite VC-dimension but finite fat-shattering dimension (Bartlett, 1998, 
Shawe-Taylor et al., 1998), such as the function classes used by AdaBoost (see, e.g., Schapire et al., 1998, Koltchinskii and Panchenko, 2002). These upper bounds provide guidance for model selection and in particular help guide data-dependent choices of regularization parameters.

To carry this agenda further, it is necessary to find general quantitative relationships between the approximation and estimation errors associated with $\phi$, and those associated with 0-1 loss. This point has been emphasized by Zhang (2003), who has presented several examples of such relationships. We simplify and extend Zhang's results, developing a general methodology for finding quantitative relationships between the risk associated with $\phi$ and the risk associated with 0-1 loss. In particular, let $R(f)$ denote the risk based on 0-1 loss and let $R^{*}=\inf _{f} R(f)$ denote the Bayes risk. Similarly, let us refer to $R_{\phi}(f)=\mathbf{E} \phi(Y f(X))$ as the " $\phi$-risk," and let $R_{\phi}^{*}=\inf _{f} R_{\phi}(f)$ denote the "optimal $\phi$-risk." We show that, for all measurable $f$,

$$
\psi\left(R(f)-R^{*}\right) \leq R_{\phi}(f)-R_{\phi}^{*}
$$

for a nondecreasing function $\psi:[0,1] \rightarrow[0, \infty)$. Moreover, we present a general variational representation of $\psi$ in terms of $\phi$, and show how this representation allows us to infer various properties of $\psi$.

This result suggests that if $\psi$ is well-behaved then minimization of $R_{\phi}(f)$ may provide a reasonable surrogate for minimization of $R(f)$. Moreover, the result provides a quantitative way to transfer assessments of statistical error in terms of "excess $\phi$-risk" $R_{\phi}(f)-R_{\phi}^{*}$ into assessments of error in terms of "excess risk" $R(f)-R^{*}$.

Although our principal goal is to understand the implications of convexity in classification, we do not impose a convexity assumption on $\phi$ at the outset. Indeed, while conditions such as convexity, continuity, and differentiability of $\phi$ are easy to verify and have natural relationships to optimization procedures, it is not immediately obvious how to relate such conditions to their statistical consequences. Thus, we consider the weakest possible condition on $\phi$ : that it is "classification-calibrated," which is essentially a pointwise form of Fisher consistency for classification (Lin, 2001). In particular, if we define $\eta(x)=P(Y=1 \mid X=x)$, then $\phi$ is classification-calibrated if, for $\eta(x) \neq 1 / 2$, the minimizer $f^{*}$ of the conditional expectation $\mathbf{E}\left[\phi\left(Y f^{*}(X)\right) \mid X=x\right]$ has the same sign as the Bayes decision rule, $\operatorname{sign}(2 \eta(x)-1)$. We show that our upper bound on excess risk in terms of excess $\phi$-risk is nontrivial precisely when $\phi$ is classification-calibrated. Obviously, no such bound is possible when $\phi$ is not classification-calibrated.

The difficulty of a pattern classification problem is closely related to the behavior of the posterior probability $\eta(X)$. In many practical problems, it is reasonable to assume that, for most $X, \eta(X)$ is not too close to $1 / 2$. Tsybakov (2001) has introduced an elegant formulation of such an assumption and considered the rate of convergence of the risk of a function that minimizes empirical risk over some fixed class $\mathcal{F}$. He showed that, under the assumption of low noise, the risk converges surprisingly quickly to the minimum over the class. If the minimum risk is nonzero, we might expect a convergence rate no faster than $1 / \sqrt{n}$. However, under Tsybakov's assumption, it can be as fast as $1 / n$. We show that minimizing empirical $\phi$-risk also leads to surprisingly fast convergence rates under this assumption. In particular, if $\phi$ is uniformly convex, the empirical $\phi$-risk converges quickly to the $\phi$-risk, and the noise assumption allows an improvement in the relationship between excess $\phi$-risk and excess risk.

These results suggest an interpretation of pattern classification methods involving a convex contrast function. It is common to view the excess risk as a combination of an estimation term and 
an approximation term:

$$
R(f)-R^{*}=\left(R(f)-\inf _{g \in \mathcal{F}} R(g)\right)+\left(\inf _{g \in \mathcal{F}} R(g)-R^{*}\right) .
$$

However, choosing a function with risk near minimal over a class $\mathcal{F}$ - that is, finding an $f$ for which the estimation term above is close to zero - is, in a minimax setting, equivalent to the problem of minimizing empirical risk, and hence is computationally infeasible for typical classes $\mathcal{F}$ of interest. Indeed, for classes typically used by boosting and kernel methods, the estimation term in this expression does not converge to zero for the minimizer of the empirical risk. On the other hand, we can also split the upper bound on excess risk into an estimation term and an approximation term:

$$
\psi\left(R(f)-R^{*}\right) \leq R_{\phi}(f)-R_{\phi}^{*}=\left(R_{\phi}(f)-\inf _{g \in \mathcal{F}} R_{\phi}(g)\right)+\left(\inf _{g \in \mathcal{F}} R_{\phi}(g)-R_{\phi}^{*}\right) .
$$

Often, it is possible to minimize $\phi$-risk efficiently. Thus, while finding an $f$ with near-minimal risk might be computationally infeasible, finding an $f$ for which this upper bound on risk is near minimal can be feasible.

The paper is organized as follows. Section 2 presents basic definitions and a statement and proof of (1). In Section 3, we introduce the convexity assumption and discuss its relationship to the other conditions. Section 4 presents a refined version of our main result in the setting of low noise. We give applications to the estimation of convergence rates in Section 5 and present our conclusions in Section 6.

\section{Relating excess risk to excess $\phi$-risk}

There are three sources of error to be considered in a statistical analysis of classification problems: the classical estimation error due to finite sample size, the classical approximation error due to the size of the function space $\mathcal{F}$, and an additional source of approximation error due to the use of a surrogate in place of the 0-1 loss function. It is this last source of error that is our focus in this section. Thus, throughout the section we (a) work with population expectations and (b) assume that $\mathcal{F}$ is the set of all measurable functions. This allows us to ignore errors due to the size of the sample and the size of the function space, and focus on the error due to the use of a surrogate for the $0-1$ loss function.

We follow the tradition in the classification literature and refer to the function $\phi$ as a loss function, since it is a function that is to be minimized to obtain a discriminant. More precisely, $\phi(Y f(X))$ is generally referred to as a "margin-based loss function," where the quantity $Y f(X)$ is known as the "margin." (It is worth noting that margin-based loss functions are rather different from distance metrics, a point that we explore in the Appendix.)

This ambiguity in the use of "loss" will not confuse; in particular, we will be careful to distinguish the risk, which is an expectation over 0-1 loss, from the " $\phi$-risk," which is an expectation over $\phi$. Our goal in this section is to relate these two quantities.

\subsection{Setup}

Let $\left(\mathcal{X} \times\{-1,1\}, \mathcal{G} \otimes 2^{\{-1,1\}}, P\right)$ be a probability space. Let $X$ be the identity function on $\mathcal{X}$ and $Y$ the identity function on $\{-1,1\}$, so that $P$ is the distribution of $(X, Y)$, i.e., for $A \in \mathcal{G} \otimes 2^{\{-1,1\}}$, 
$P((X, Y) \in A)=P(A)$. Let $P_{X}$ on $(\mathcal{X}, \mathcal{G})$ be the marginal distribution of $X$, and let $\eta: \mathcal{X} \rightarrow[0,1]$ be a measurable function such that $\eta(X)$ is a version of $P(Y=1 \mid X)$. Throughout this section, $f$ is understood as a measurable mapping from $\mathcal{X}$ into $\mathbb{R}$.

Define the $\{0,1\}$-risk, or just risk, of $f$ as

$$
R(f)=P(\operatorname{sign}(f(X)) \neq Y),
$$

where $\operatorname{sign}(\alpha)=1$ for $\alpha>0$ and -1 otherwise. (The particular choice of the value of $\operatorname{sign}(0)$ is not important, but we need to fix some value in $\{ \pm 1\}$ for the definitions that follow.) Based on an i.i.d. sample $D_{n}=\left(\left(X_{1}, Y_{1}\right), \ldots,\left(X_{n}, Y_{n}\right)\right)$, we want to choose a function $f_{n}$ with small risk.

Define the Bayes risk $R^{*}=\inf _{f} R(f)$, where the infimum is over all measurable $f$. Then any $f$ satisfying $\operatorname{sign}(f(X))=\operatorname{sign}(\eta(X)-1 / 2)$ a.s. on $\{\eta(X) \neq 1 / 2\}$ has $R(f)=R^{*}$.

Fix a function $\phi: \mathbb{R} \rightarrow[0, \infty)$. Define the $\phi$-risk of $f$ as

$$
R_{\phi}(f)=\mathbf{E} \phi(Y f(X)) .
$$

Let $\mathcal{F}$ be a class of functions $f: \mathcal{X} \rightarrow \mathbb{R}$. Let $f_{n}=\hat{f}_{\phi}$ be a function in $\mathcal{F}$ which minimizes the empirical expectation of $\phi(Y f(X))$,

$$
\hat{R}_{\phi}(f)=\hat{\mathbf{E}} \phi(Y f(X))=\frac{1}{n} \sum_{i=1}^{n} \phi\left(Y_{i} f\left(X_{i}\right)\right) .
$$

Thus we treat $\phi$ as specifying a contrast function that is to be minimized in determining the discriminant function $f_{n}$.

\subsection{Basic conditions on the loss function}

For (almost all) $x$, we define the conditional $\phi$-risk

$$
\mathbf{E}(\phi(Y f(X)) \mid X=x)=\eta(x) \phi(f(x))+(1-\eta(x)) \phi(-f(x)) .
$$

It is useful to think of the conditional $\phi$-risk in terms of a generic conditional probability $\eta \in[0,1]$ and a generic classifier value $\alpha \in \mathbb{R}$. To express this viewpoint, we introduce the generic conditional $\phi$-risk

$$
C_{\eta}(\alpha)=\eta \phi(\alpha)+(1-\eta) \phi(-\alpha) .
$$

The notation suppresses the dependence on $\phi$. The generic conditional $\phi$-risk coincides with the conditional $\phi$-risk of $f$ at $x \in \mathcal{X}$ if we take $\eta=\eta(x)$ and $\alpha=f(x)$. Here, varying $\alpha$ in the generic formulation corresponds to varying $f$ in the original formulation, for fixed $x$.

For $\eta \in[0,1]$, define the optimal conditional $\phi$-risk

$$
H(\eta)=\inf _{\alpha \in \mathbb{R}} C_{\eta}(\alpha)=\inf _{\alpha \in \mathbb{R}}(\eta \phi(\alpha)+(1-\eta) \phi(-\alpha)) .
$$

Then the optimal $\phi$-risk satisfies

$$
R_{\phi}^{*}:=\inf _{f} R_{\phi}(f)=\mathbf{E} H(\eta(X)),
$$

where the infimum is over measurable functions. 
We say that a sequence $\alpha_{1}, \alpha_{2}, \ldots$ achieves $H$ at $\eta$ if

$$
\lim _{i \rightarrow \infty} C_{\eta}\left(\alpha_{i}\right)=\lim _{i \rightarrow \infty}\left(\eta \phi\left(\alpha_{i}\right)+(1-\eta) \phi\left(-\alpha_{i}\right)\right)=H(\eta)
$$

If the infimum in the definition of $H(\eta)$ is uniquely attained for some $\alpha$, we can define $\alpha^{*}:[0,1] \rightarrow \mathbb{R}$ by

$$
\alpha^{*}(\eta)=\underset{\alpha \in \mathbb{R}}{\arg \min } C_{\eta}(\alpha)=\underset{\alpha \in \mathbb{R}}{\arg \min } \eta \phi(\alpha)+(1-\eta) \phi(-\alpha) .
$$

In that case, we define $f_{\phi}^{*}: \mathcal{X} \rightarrow \mathbb{R}$, up to $P_{X}$-null sets, by

$$
\begin{aligned}
f_{\phi}^{*}(x) & =\underset{\alpha \in \mathbb{R}}{\arg \min } \mathbf{E}(\phi(Y \alpha) \mid X=x) \\
& =\alpha^{*}(\eta(x))
\end{aligned}
$$

and then

$$
R_{\phi}\left(f_{\phi}^{*}\right)=\mathbf{E} H(\eta(X))=R_{\phi}^{*}
$$

For $\eta \in[0,1]$, define

$$
H^{-}(\eta)=\inf _{\alpha: \alpha(2 \eta-1) \leq 0} C_{\eta}(\alpha)=\inf _{\alpha: \alpha(2 \eta-1) \leq 0}(\eta \phi(\alpha)+(1-\eta) \phi(-\alpha)) .
$$

This is the optimal value of the conditional $\phi$-risk, under the constraint that the sign of the argument $\alpha$ disagrees with that of $2 \eta-1$.

We now turn to the basic condition we impose on $\phi$. This condition generalizes the requirement that the minimizer of $C_{\eta}(\alpha)$ (if it exists) has the correct sign. This is a minimal condition that can be viewed as a pointwise form of Fisher consistency for classification.

Definition 1. We say that $\phi$ is classification-calibrated if, for any $\eta \neq 1 / 2$,

$$
H^{-}(\eta)>H(\eta)
$$

Equivalently, $\phi$ is classification-calibrated if any sequence $\alpha_{1}, \alpha_{2}, \ldots$ that achieves $H$ at $\eta$ satisfies $\liminf _{i \rightarrow \infty} \operatorname{sign}\left(\alpha_{i}(\eta-1 / 2)\right)=1$. Since $\operatorname{sign}\left(\alpha_{i}(\eta-1 / 2)\right) \in\{-1,1\}$, this is equivalent to the requirement $\lim _{i \rightarrow \infty} \operatorname{sign}\left(\alpha_{i}(\eta-1 / 2)\right)=1$, or simply that $\operatorname{sign}\left(\alpha_{i}(\eta-1 / 2)\right) \neq 1$ only finitely often.

\subsection{The $\psi$-transform and the relationship between excess risks}

We begin by defining a functional transform of the loss function:

Definition 2. We define the $\psi$-transform of a loss function as follows. Given $\phi: \mathbb{R} \rightarrow[0, \infty)$, define the function $\psi:[0,1] \rightarrow[0, \infty)$ by $\psi=\tilde{\psi}^{* *}$, where

$$
\tilde{\psi}(\theta)=H^{-}\left(\frac{1+\theta}{2}\right)-H\left(\frac{1+\theta}{2}\right),
$$

and $g^{* *}:[0,1] \rightarrow \mathbb{R}$ is the Fenchel-Legendre biconjugate of $g:[0,1] \rightarrow \mathbb{R}$, which is characterized by

$$
\text { epi } g^{* *}=\overline{\mathrm{co}} \text { epi } g \text {. }
$$

Here $\overline{\text { co }} S$ is the closure of the convex hull of the set $S$, and epi $g$ is the epigraph of the function $g$, that is, the set $\{(x, t): x \in[0,1], g(x) \leq t\}$. The nonnegativity of $\psi$ is established below in Lemma 5 , part 7 . 
Recall that $g$ is convex if and only if epi $g$ is a convex set, and $g$ is closed (epi $g$ is a closed set) if and only if $g$ is lower semicontinuous (Rockafellar, 1997). By Lemma 5, part 5, $\tilde{\psi}$ is continuous, so in fact the closure operation in Definition 2 is vacuous. We therefore have that $\psi$ is simply the functional convex hull of $\tilde{\psi}$,

$$
\psi=\operatorname{co} \tilde{\psi},
$$

which is equivalent to the epigraph convex hull condition of the definition. This implies that $\psi=\tilde{\psi}$ if and only if $\tilde{\psi}$ is convex; see Example 5 for a loss function where the latter fails.

The importance of the $\psi$-transform is shown by the following theorem.

Theorem 3. 1. For any nonnegative loss function $\phi$, any measurable $f: \mathcal{X} \rightarrow \mathbb{R}$ and any probability distribution on $\mathcal{X} \times\{ \pm 1\}$,

$$
\psi\left(R(f)-R^{*}\right) \leq R_{\phi}(f)-R_{\phi}^{*} .
$$

2. Suppose $|\mathcal{X}| \geq 2$. For any nonnegative loss function $\phi$, any $\epsilon>0$ and any $\theta \in[0,1]$, there is a probability distribution on $\mathcal{X} \times\{ \pm 1\}$ and a function $f: \mathcal{X} \rightarrow \mathbb{R}$ such that

$$
R(f)-R^{*}=\theta
$$

and

$$
\psi(\theta) \leq R_{\phi}(f)-R_{\phi}^{*} \leq \psi(\theta)+\epsilon .
$$

3. The following conditions are equivalent.

(a) $\phi$ is classification-calibrated.

(b) For any sequence $\left(\theta_{i}\right)$ in $[0,1]$,

$$
\psi\left(\theta_{i}\right) \rightarrow 0 \text { if and only if } \theta_{i} \rightarrow 0 .
$$

(c) For every sequence of measurable functions $f_{i}: \mathcal{X} \rightarrow \mathbb{R}$ and every probability distribution on $\mathcal{X} \times\{ \pm 1\}$

$$
R_{\phi}\left(f_{i}\right) \rightarrow R_{\phi}^{*} \quad \text { implies } \quad R\left(f_{i}\right) \rightarrow R^{*} .
$$

Here we mention that classification-calibration implies $\psi$ is invertible on $[0,1]$, so in that case it is meaningful to write the upper bound on excess risk in Theorem 3(1) as $\psi^{-1}\left(R_{\phi}(f)-R_{\phi}^{*}\right)$. Invertibility follows from convexity of $\psi$ together with Lemma 5 , parts 6,8 , and 9 .

Zhang (2003) has given a comparison theorem like Parts 1 and $3 \mathrm{~b}$ of this theorem, for convex $\phi$ that satisfy certain conditions. These conditions imply an assumption on the rate of growth (and convexity) of $\tilde{\psi}$. Lugosi and Vayatis (2003) show that a limiting result like Part 3c holds for strictly convex, differentiable, monotonic $\phi$. In Section 3, we show that if $\phi$ is convex, classificationcalibration is equivalent to a simple derivative condition on $\phi$ at zero. Clearly, the conclusions of Theorem 3 hold under weaker conditions than those assumed by Zhang (2003) or Lugosi and Vayatis (2003). Steinwart (2002) has shown that if $\phi$ is continuous and classification-calibrated, then $R_{\phi}\left(f_{i}\right) \rightarrow R_{\phi}^{*}$ implies $R\left(f_{i}\right) \rightarrow R^{*}$. Theorem 3 shows that we may obtain a more quantitative statement of the relationship between these excess risks, under weaker conditions.

Before presenting the proof of Theorem 3, we illustrate the $\psi$-transform in the case of four commonly used margin-based loss functions. 

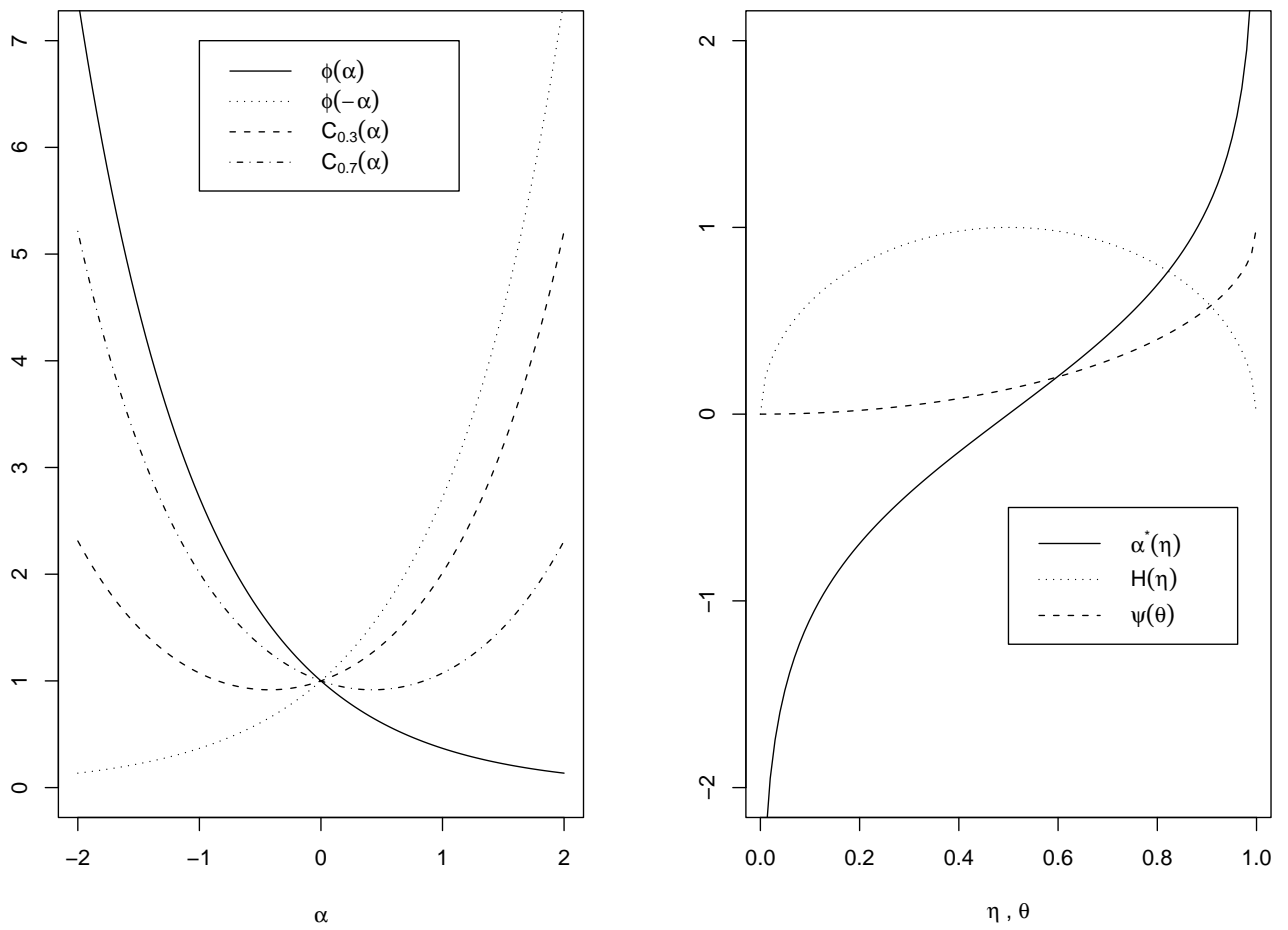

Figure 2: Exponential loss. The left panel shows $\phi(\alpha)$, its reflection $\phi(-\alpha)$, and two different convex combinations of these functions, for $\eta=0.3$ and $\eta=0.7$. Note that the minima of these combinations are the values $H(\eta)$, and the minimizing arguments are the values $\alpha^{*}(\eta)$. The right panel shows $H(\eta)$ and $\alpha^{*}(\eta)$ plotted as a function of $\eta$, and the $\psi$-transform $\psi(\theta)$.

Example 1 (Exponential loss). Here $\phi(\alpha)=\exp (-\alpha)$. Figure 2, left panel, shows $\phi(\alpha), \phi(-\alpha)$, and the generic conditional $\phi$-risk $C_{\eta}(\alpha)$ for $\eta=0.3$ and $\eta=0.7$. In this case, $\phi$ is strictly convex on $\mathbb{R}$, hence $C_{\eta}(\alpha)$ is also strictly convex on $\mathbb{R}$, for every $\eta$. So $C_{\eta}$ is either minimal at a unique stationary point, or it attains no minimum. Indeed, if $\eta=0$, then $C_{\eta}(\alpha) \rightarrow 0$ as $\alpha \rightarrow-\infty$; if $\eta=1$, then $C_{\eta}(\alpha) \rightarrow 0$ as $\alpha \rightarrow \infty$. Thus we have $H(0)=H(1)=0$ for exponential loss. For $\eta \in(0,1)$, solving for the stationary point yields the unique minimizer

$$
\alpha^{*}(\eta)=\frac{1}{2} \log \left(\frac{\eta}{1-\eta}\right) .
$$

We may then simplify the identity $H(\eta)=C_{\eta}\left(\alpha^{*}(\eta)\right)$ to obtain

$$
H(\eta)=2 \sqrt{\eta(1-\eta)} .
$$

Notice that this expression is correct even for $\eta$ equal to 0 or 1 . It is easy to check that

$$
H^{-}\left(\frac{1+\theta}{2}\right) \equiv \exp (0)=1
$$



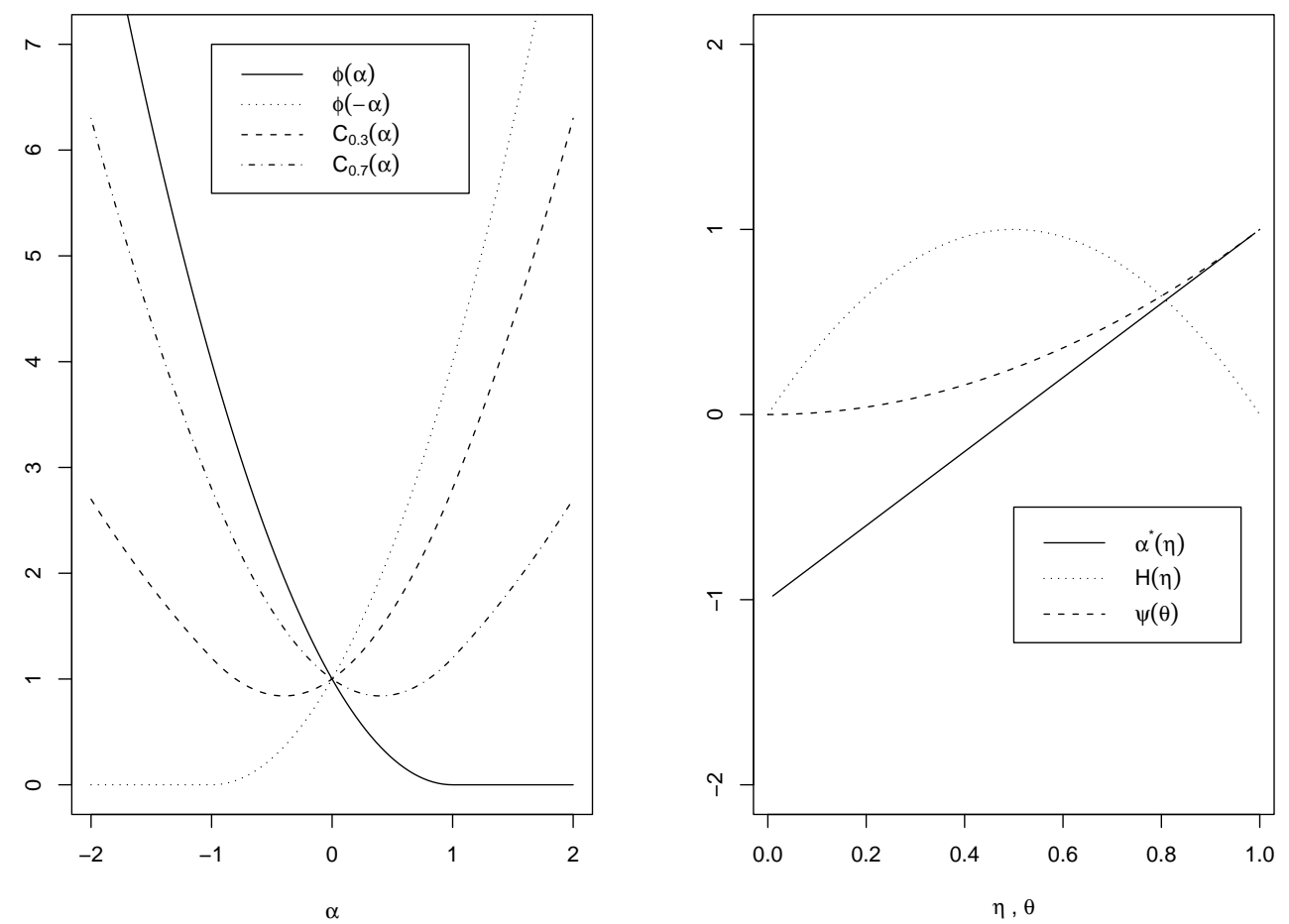

Figure 3: Truncated quadratic loss.

and so

$$
\tilde{\psi}(\theta)=1-\sqrt{1-\theta^{2}} .
$$

Since $\tilde{\psi}$ is convex, $\psi=\tilde{\psi}$. The right panel of Figure 2 shows the graphs of $\alpha^{*}, H$, and $\psi$ over the interval $[0,1]$.

Finally, for $0<\eta<1, \operatorname{sign}\left(\alpha^{*}(\eta)\right)=\operatorname{sign}(\eta-1 / 2)$ by inspection. Also, a sequence $\left(\alpha_{i}\right)$ can achieve $H$ at $\eta=0$ (respectively, 1 ) only if it diverges to $-\infty$ (respectively, $\infty$ ). It therefore follows that exponential loss is classification-calibrated.

Example 2 (Truncated quadratic loss). Now consider $\phi(\alpha)=[\max \{1-\alpha, 0\}]^{2}$, as depicted together with $\phi(-\alpha), C_{0.3}(\alpha)$, and $C_{0.7}(\alpha)$ in the left panel of Figure 3 . If $\eta=0$, it is clear that any $\alpha \in(-\infty,-1]$ makes $C_{\eta}(\alpha)$ vanish. Similarly, any $\alpha \in[1, \infty)$ makes the conditional $\phi$-risk vanish when $\eta=1$. On the other hand, when $0<\eta<1, C_{\eta}$ is strictly convex with a (unique) stationary point, and solving for it yields

$$
\alpha^{*}(\eta)=2 \eta-1 \text {. }
$$

Notice that, though $\alpha^{*}$ is in principle undefined at 0 and 1 , we could choose to fix $\alpha^{*}(0)=-1$ and $\alpha^{*}(1)=1$, which are valid settings. This would extend $(2)$ to all of $[0,1]$.

As in Example 1, we may simplify the identity $H(\eta)=C_{\eta}\left(\alpha^{*}(\eta)\right)$ for $0<\eta<1$ to obtain

$$
H(\eta)=4 \eta(1-\eta)
$$



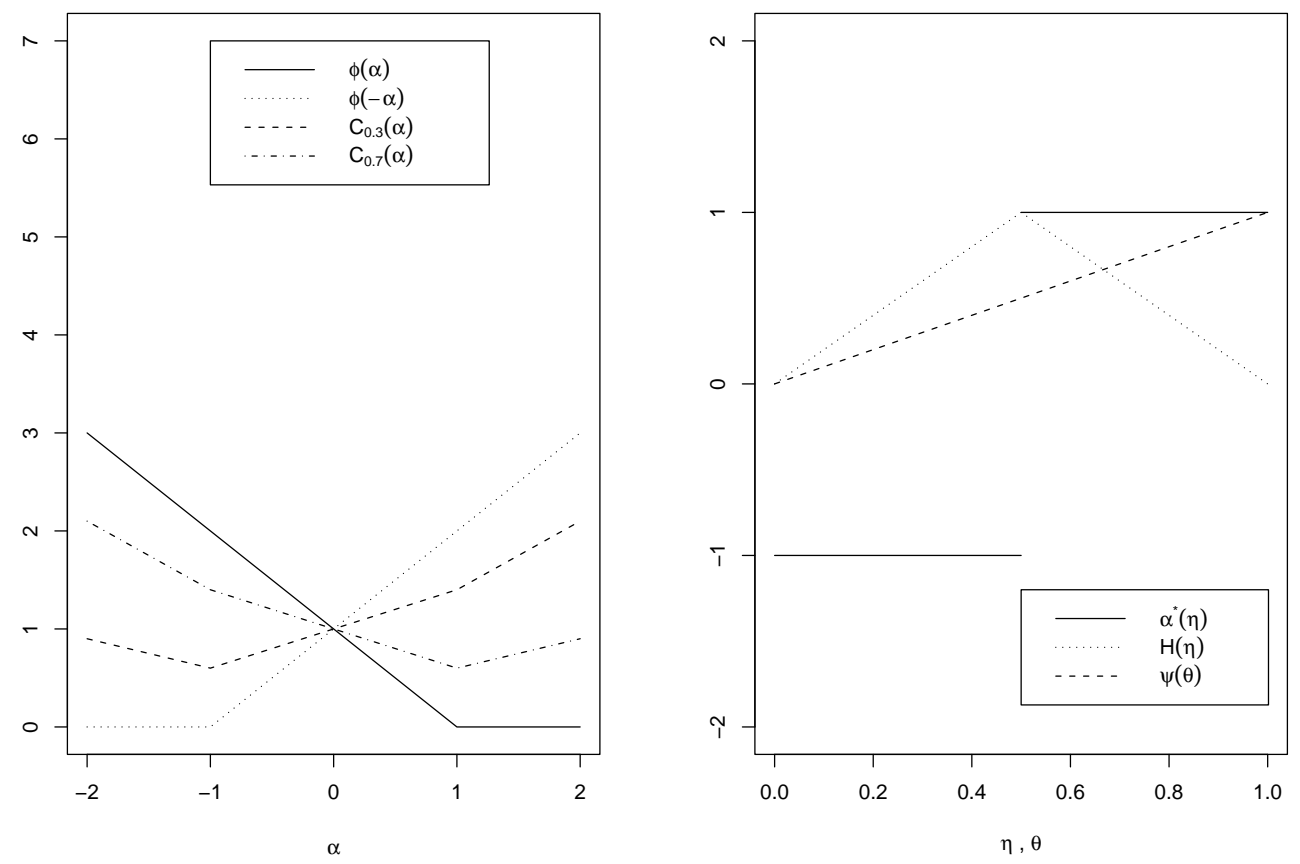

Figure 4: Hinge loss.

which is also correct for $\eta=0$ and 1 , as noted. It is also immediate that $H^{-}((1+\theta) / 2) \equiv \phi(0)=1$, so we have

$$
\tilde{\psi}(\theta)=\theta^{2} .
$$

Again, $\tilde{\psi}$ is convex, so $\psi=\tilde{\psi}$. The right panel of Figure 3 shows $\alpha^{*}, H$, and $\psi$. Observe that truncated quadratic loss is classification-calibrated: the case $0<\eta<1$ is obvious from (2); for $\eta=0$ or 1 , it follows because any $\left(\alpha_{i}\right)$ achieving $H$ at 0 (respectively, 1 ) must eventually take values in $(-\infty,-1]$ (respectively, $[1, \infty))$.

Example 3 (Hinge loss). Here we take $\phi(\alpha)=\max \{1-\alpha, 0\}$, which is shown in the left panel of Figure 4 along with $\phi(-\alpha), C_{0.3}(\alpha)$, and $C_{0.7}(\alpha)$. By direct consideration of the piecewise-linear form of $C_{\eta}(\alpha)$, it is easy to see that for $\eta=0$, each $\alpha \leq-1$ makes $C_{\eta}(\alpha)$ vanish, just as in Example 2. The same holds for $\alpha \geq 1$ when $\eta=1$. Now for $\eta \in(0,1)$, we see that $C_{\eta}$ decreases strictly on $(-\infty,-1]$ and increases strictly on $[1, \infty)$. Thus any minima must lie in $[-1,1]$. But $C_{\eta}$ is linear on $[-1,1]$, so the minimum must be attained at 1 for $\eta>1 / 2,-1$ for $\eta<1 / 2$, and anywhere in $[-1,1]$ for $\eta=1 / 2$. We have argued that

$$
\alpha^{*}(\eta)=\operatorname{sign}(\eta-1 / 2)
$$

for all $\eta \in(0,1)$ other than $1 / 2$. Since (3) yields valid minima at $0,1 / 2$, and 1 also, we could choose to extend it to the whole unit interval. Regardless, a simple direct verification as in the previous examples shows

$$
H(\eta)=2 \min \{\eta, 1-\eta\}
$$



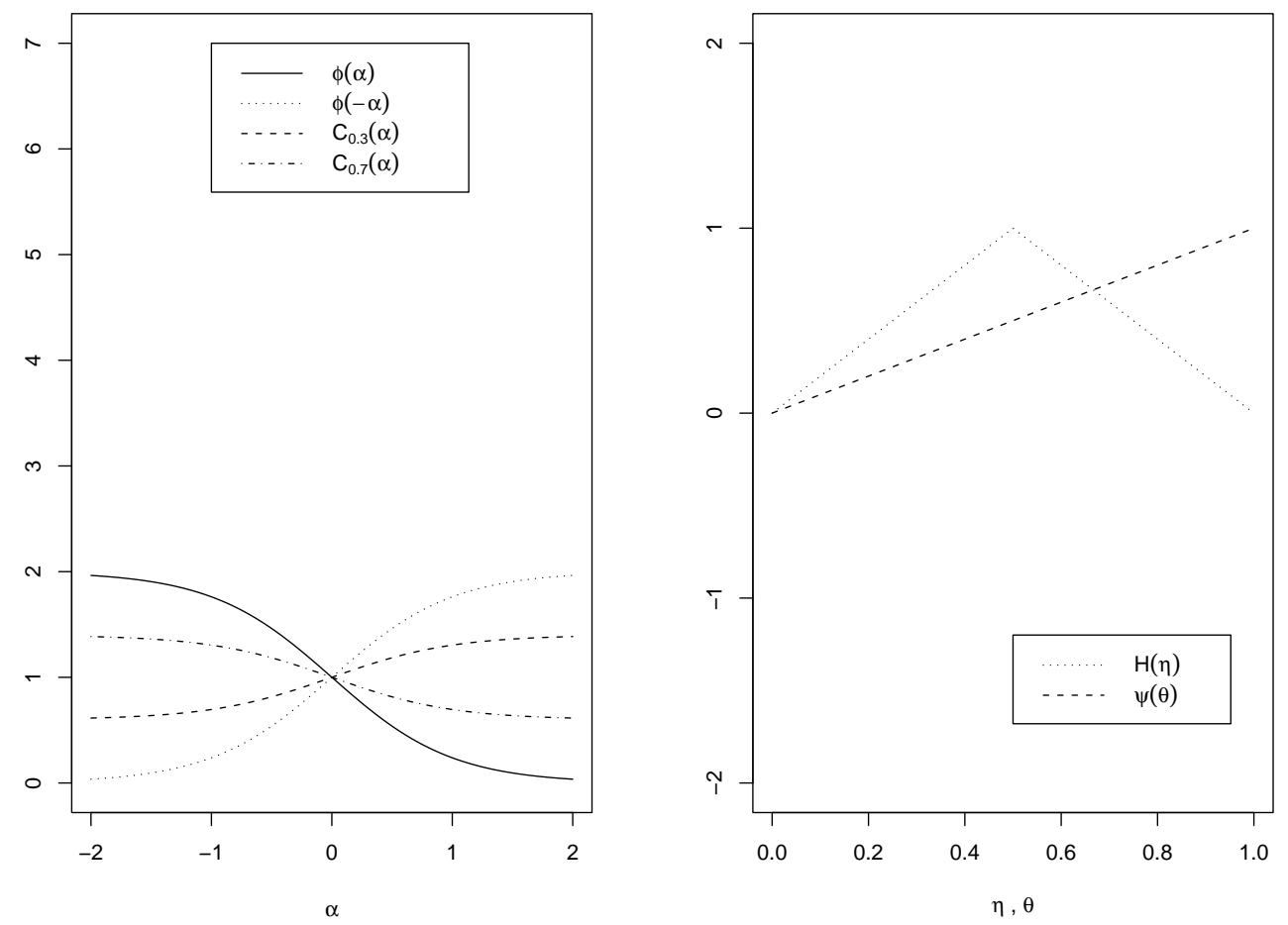

Figure 5: Sigmoid loss.

for $0 \leq \eta \leq 1$. Since $H^{-}((1+\theta) / 2) \equiv \phi(0)=1$, we have

$$
\tilde{\psi}(\theta)=\theta
$$

and $\psi=\tilde{\psi}$ by convexity. We present $\alpha^{*}, H$, and $\psi$ in the right panel of Figure 4 . To conclude, notice that the form of (3) and separate considerations for $\eta \in\{0,1\}$, as in Example 2, easily imply that hinge loss is classification-calibrated.

Example 4 (Sigmoid loss). We conclude by examining a non-convex loss function. Let $\phi(\alpha)=$ $1-\tanh (k \alpha)$ for some fixed $k>0$. Figure 5 , left panel, depicts $\phi(\alpha)$ with $k=1$, as well as $\phi(-\alpha)$, $C_{0.3}(\alpha)$, and $C_{0.7}(\alpha)$. Using the fact that tanh is an odd function, we can rewrite the conditional $\phi$-risk as

$$
C_{\eta}(\alpha)=1+(1-2 \eta) \tanh (k \alpha) .
$$

From this expression, two facts are clear. First, when $\eta=1 / 2$, every $\alpha$ minimizes $C_{\eta}(\alpha)$, because it is identically 1 . Second, when $\eta \neq 1 / 2, C_{\eta}(\alpha)$ attains no minimum, because tanh has no maximal or minimal value on $\mathbb{R}$. Hence $\alpha^{*}$ is not defined for any $\eta$.

Inspecting (4), for $0 \leq \eta<1 / 2$ we obtain $H(\eta)=2 \eta$ by letting $\alpha \rightarrow-\infty$. Analogously, when $\alpha \rightarrow \infty$, we get $H(\eta)=2(1-\eta)$ for $1 / 2<\eta \leq 1$. Thus we have

$$
H(\eta)=2 \min \{\eta, 1-\eta\}, \quad 0 \leq \eta \leq 1 .
$$


Since $H^{-}((1+\theta) / 2) \equiv \phi(0)=1$, we have

$$
\tilde{\psi}(\theta)=\theta
$$

and convexity once more gives $\psi=\tilde{\psi}$. We present $H$ and $\psi$ in the right panel of Figure 5 . Finally, the foregoing considerations imply that sigmoid loss is classification-calibrated, provided we note carefully that the definition of classification-calibration requires nothing when $\eta=1 / 2$.

\subsection{Properties of $\psi$ and proof of Theorem 3}

The following elementary lemma will be useful throughout the paper.

Lemma 4. Suppose $g: \mathbb{R} \rightarrow \mathbb{R}$ is convex and $g(0)=0$. Then

1. for all $\lambda \in[0,1]$ and $x \in \mathbb{R}$,

$$
g(\lambda x) \leq \lambda g(x)
$$

2. for all $x>0,0 \leq y \leq x$,

$$
g(y) \leq \frac{y}{x} g(x)
$$

3. $g(x) / x$ is increasing on $(0, \infty)$.

Proof. For $1, g(\lambda x)=g(\lambda x+(1-\lambda) 0) \leq \lambda g(x)+(1-\lambda) g(0)=\lambda g(x)$. To see 2 , put $\lambda=y / x$ in 1 . For 3, rewrite 2 as $g(y) / y \leq g(x) / x$.

Lemma 5. The functions $H, H^{-}$and $\psi$ have the following properties:

1. $H$ and $H^{-}$are symmetric about $1 / 2$ : for all $\eta \in[0,1], H(\eta)=H(1-\eta), H^{-}(\eta)=H^{-}(1-\eta)$.

2. $H$ is concave and, for $0 \leq \eta \leq 1$, it satisfies

$$
H(\eta) \leq H\left(\frac{1}{2}\right)=H^{-}\left(\frac{1}{2}\right)
$$

3. If $\phi$ is classification-calibrated, then $H(\eta)<H(1 / 2)$ for all $\eta \neq 1 / 2$.

4. $H^{-}$is concave on $[0,1 / 2]$ and on $[1 / 2,1]$, and for $0 \leq \eta \leq 1$ it satisfies

$$
H^{-}(\eta) \geq H(\eta)
$$

5. $H, H^{-}$and $\tilde{\psi}$ are continuous on $[0,1]$.

6. $\psi$ is continuous on $[0,1]$.

7. $\psi$ is nonnegative and minimal at 0 .

8. $\psi(0)=0$.

9. The following statements are equivalent:

(a) $\phi$ is classification-calibrated. 


$$
\text { (b) } \psi(\theta)>0 \text { for all } \theta \in(0,1] \text {. }
$$

Before proving the lemma, we point out that there is no converse to part 3 . To see this, let $\phi$ be classification-calibrated, and consider the loss function $\tilde{\phi}(\alpha)=\phi(-\alpha)$, with corresponding $\tilde{H}(\eta)$. Since $\left(\alpha_{i}\right)$ achieves $H$ at $\eta$ if and only if $\left(-\alpha_{i}\right)$ achieves $\tilde{H}$ at $\eta$, we see that $\tilde{\phi}$ is not classification-calibrated. However, $\tilde{H}(\eta)=H(1-\eta)$, so because part 3 holds for $\phi$, it must also hold for $\tilde{\phi}$.

Proof. 1 is immediate from the definitions.

For 2, concavity follows because $H$ is an infimum of concave (affine) functions of $\eta$. Now, since $H$ is concave and symmetric about $1 / 2, H(1 / 2)=H((1 / 2) \eta+(1 / 2)(1-\eta)) \geq(1 / 2) H(\eta)+$ $(1 / 2) H(1-\eta)=H(\eta)$. Thus $H$ is maximal at $1 / 2$. To see that $H(1 / 2)=H^{-}(1 / 2)$, notice that $\alpha(2 \eta-1) \leq 0$ for all $\alpha$ when $\eta=1 / 2$.

To prove 3 , assume that there is an $\eta \neq 1 / 2$ with $H(\eta)=H(1 / 2)$. Fix a sequence $\alpha_{1}, \alpha_{2}, \ldots$ that achieves $H$ at $1 / 2$. By the assumption,

$$
\liminf _{i \rightarrow \infty}\left(\eta \phi\left(\alpha_{i}\right)+(1-\eta) \phi\left(-\alpha_{i}\right)\right) \geq H(\eta)=H(1 / 2)=\lim _{i \rightarrow \infty} \frac{\phi\left(\alpha_{i}\right)+\phi\left(-\alpha_{i}\right)}{2},
$$

Rearranging, we have

$$
(\eta-1 / 2) \liminf _{i \rightarrow \infty}\left(\phi\left(\alpha_{i}\right)-\phi\left(-\alpha_{i}\right)\right) \geq 0
$$

Since $H(1-\eta)=H(\eta)$, the same argument shows that $H(\eta)=H(1 / 2)$ implies

$$
(\eta-1 / 2) \liminf _{i \rightarrow \infty}\left(\phi\left(-\alpha_{i}\right)-\phi\left(\alpha_{i}\right)\right) \geq 0
$$

It follows that

$$
\lim _{i \rightarrow \infty}\left(\phi\left(\alpha_{i}\right)-\phi\left(-\alpha_{i}\right)\right)=0,
$$

so all the expressions in (5) are equal. Hence, $H$ is achieved by $\left(\alpha_{i}\right)$ at $\eta$, and if $\phi$ is classificationcalibrated we must have that

$$
\liminf _{i \rightarrow \infty}\left(\operatorname{sign}\left(\alpha_{i}(\eta-1 / 2)\right)=1 .\right.
$$

The same argument shows that $H$ is achieved by $\left(\alpha_{i}\right)$ at $1-\eta$, and if $\phi$ is classification-calibrated we must have that

$$
\limsup _{i \rightarrow \infty}\left(\operatorname{sign}\left(\alpha_{i}(\eta-1 / 2)\right)=-1 .\right.
$$

Thus, if $H(\eta)=H(1 / 2), \phi$ is not classification-calibrated.

For $4, H^{-}$is concave on $[0,1 / 2]$ by the same argument as for the concavity of $H$. (Notice that when $\eta<1 / 2, H^{-}$is an infimum over a set of concave functions, but in this case when $\eta>1 / 2$, it is an infimum over a different set of concave functions.) The inequality $H^{-} \geq H$ follows from the definitions.

For 5, first notice that the concavity of $H$ implies that it is continuous on the relative interior of its domain, i.e. $(0,1)$. Thus, to show that $H$ is continuous $[0,1]$, it suffices (by symmetry) to show that it is left continuous at 1 . Because $[0,1]$ is locally simplicial in the sense of Rockafellar (1997), his Theorem 10.2 gives lower semicontinuity of $H$ at 1 (equivalently, upper semicontinuity of the convex function $-H$ at 1). To see upper semicontinuity of $H$ at 1 , on the other hand, fix 
any $\epsilon>0$ and choose $\alpha_{\epsilon}$ such that $\phi\left(\alpha_{\epsilon}\right) \leq H(1)+\epsilon / 2$. Then for any $\eta$ between $1-\epsilon /\left(2 \phi\left(-\alpha_{\epsilon}\right)\right)$ and 1 we have

$$
H(\eta) \leq C_{\eta}\left(\alpha_{\epsilon}\right) \leq H(1)+\epsilon
$$

Since this is true for any $\epsilon, \lim \sup _{\eta \rightarrow 1} H(\eta) \leq H(1)$, which is upper semicontinuity. Thus $H$ is left continuous at 1 . The same argument shows that $H^{-}$is continuous on $(0,1 / 2)$ and $(1 / 2,1)$, and left continuous at $1 / 2$ and 1 . Symmetry implies that $H^{-}$is continuous on the closed interval $[0,1]$. The continuity of $\tilde{\psi}$ is now immediate.

To see 6 , observe that $\psi$ is a closed convex function with locally simplicial domain $[0,1]$, so its continuity follows by once again applying Theorem 10.2 of Rockafellar (1997).

It follows immediately from 2 and 4 that $\tilde{\psi}$ is nonnegative and minimal at 0 . Since epi $\psi$ is the convex hull of epi $\tilde{\psi}$, i.e., the set of all convex combinations of points in epi $\tilde{\psi}$, we see that $\psi$ is also nonnegative and minimal at 0 , which is 7 .

8 follows immediately from 2 .

To prove 9, suppose first that $\phi$ is classification-calibrated. Then for all $\theta \in(0,1], \tilde{\psi}(\theta)>0$. But every point in epi $\psi$ is a convex combination of points in epi $\tilde{\psi}$, so if $(\theta, 0) \in$ epi $\psi$, we can only have $\theta=0$. Hence for $\theta \in(0,1]$, points in epi $\psi$ of the form $(\theta, c)$ must have $c>0$, and closure of $\tilde{\psi}$ now implies $\psi(\theta)>0$. For the converse, notice that if $\phi$ is not classification-calibrated, then some $\theta>0$ has $\tilde{\psi}(\theta)=0$, and so $\psi(\theta)=0$.

Proof. (Of Theorem 3). For Part 1, it is straightforward to show that

$$
\begin{aligned}
R(f)-R^{*} & =R(f)-R(\eta-1 / 2) \\
& =\mathbf{E}(\mathbf{1}[\operatorname{sign}(f(X)) \neq \operatorname{sign}(\eta(X)-1 / 2)]|2 \eta(X)-1|),
\end{aligned}
$$

where $\mathbf{1}[\Phi]$ is 1 if the predicate $\Phi$ is true and 0 otherwise (see, for example, Devroye et al., 1996). We can apply Jensen's inequality, since $\psi$ is convex by definition, and the fact that $\psi(0)=0$ (Lemma 5, part 8) to show that

$$
\begin{aligned}
\psi\left(R(f)-R^{*}\right) & \leq \mathbf{E} \psi(\mathbf{1}[\operatorname{sign}(f(X)) \neq \operatorname{sign}(\eta(X)-1 / 2)]|2 \eta(X)-1|) \\
& =\mathbf{E}(\mathbf{1}[\operatorname{sign}(f(X)) \neq \operatorname{sign}(\eta(X)-1 / 2)] \psi(|2 \eta(X)-1|)) .
\end{aligned}
$$

Now, from the definition of $\psi$ we know that $\psi(\theta) \leq \tilde{\psi}(\theta)$, so we have

$$
\begin{aligned}
\psi\left(R(f)-R^{*}\right) & \leq \mathbf{E}(\mathbf{1}[\operatorname{sign}(f(X)) \neq \operatorname{sign}(\eta(X)-1 / 2)] \tilde{\psi}(|2 \eta(X)-1|)) \\
& =\mathbf{E}\left(\mathbf{1}[\operatorname{sign}(f(X)) \neq \operatorname{sign}(\eta(X)-1 / 2)]\left(H^{-}(\eta(X))-H(\eta(X))\right)\right) \\
& =\mathbf{E}\left(\mathbf{1}[\operatorname{sign}(f(X)) \neq \operatorname{sign}(\eta(X)-1 / 2)]\left(\inf _{\alpha: \alpha(2 \eta(X)-1) \leq 0} C_{\eta(X)}(\alpha)-H(\eta(X))\right)\right) \\
& \leq \mathbf{E}\left(C_{\eta(X)}(f(X))-H(\eta(X))\right) \\
& =R_{\phi}(f)-R_{\phi}^{*},
\end{aligned}
$$

where we have used the fact that for any $x$, and in particular when $\operatorname{sign}(f(x))=\operatorname{sign}(\eta(x)-1 / 2)$, we have $C_{\eta(x)}(f(x)) \geq H(\eta(x))$.

For Part 2, the first inequality is from Part 1. For the second, fix $\epsilon>0$ and $\theta \in[0,1]$. From the definition of $\psi$, we can choose $\gamma, \alpha_{1}, \alpha_{2} \in[0,1]$ for which $\theta=\gamma \alpha_{1}+(1-\gamma) \alpha_{2}$ and 
$\psi(\theta) \geq \gamma \tilde{\psi}\left(\alpha_{1}\right)+(1-\gamma) \tilde{\psi}\left(\alpha_{2}\right)-\epsilon / 2$. Choose distinct $x_{1}, x_{2} \in \mathcal{X}$, and choose $P_{X}$ such that $P_{X}\left\{x_{1}\right\}=\gamma, P_{X}\left\{x_{2}\right\}=1-\gamma, \eta\left(x_{1}\right)=\left(1+\alpha_{1}\right) / 2$, and $\eta\left(x_{2}\right)=\left(1+\alpha_{2}\right) / 2$. From the definition of $H^{-}$, we can choose $f: \mathcal{X} \rightarrow \mathbb{R}$ such that $f\left(x_{1}\right) \leq 0, f\left(x_{2}\right) \leq 0, C_{\eta\left(x_{1}\right)}\left(f\left(x_{1}\right)\right) \leq H^{-}\left(\eta\left(x_{1}\right)\right)+\epsilon / 2$ and $C_{\eta\left(x_{2}\right)}\left(f\left(x_{2}\right)\right) \leq H^{-}\left(\eta\left(x_{2}\right)\right)+\epsilon / 2$. Then we have

$$
\begin{aligned}
R_{\phi}(f)-R_{\phi}^{*} & =\mathbf{E} \phi(Y f(X))-\inf _{g} \mathbf{E} \phi(Y g(X)) \\
& =\gamma\left(C_{\eta\left(x_{1}\right)}\left(f\left(x_{1}\right)\right)-H\left(\eta\left(x_{1}\right)\right)\right)+(1-\gamma)\left(C_{\eta\left(x_{2}\right)}\left(f\left(x_{2}\right)\right)-H\left(\eta\left(x_{2}\right)\right)\right) \\
& \leq \gamma\left(H^{-}\left(\eta\left(x_{1}\right)\right)-H\left(\eta\left(x_{1}\right)\right)\right)+(1-\gamma)\left(H^{-}\left(\eta\left(x_{2}\right)\right)-H\left(\eta\left(x_{2}\right)\right)\right)+\epsilon / 2 \\
& =\gamma \tilde{\psi}\left(\alpha_{1}\right)+(1-\gamma) \tilde{\psi}\left(\alpha_{2}\right)+\epsilon / 2 \\
& \leq \psi(\theta)+\epsilon .
\end{aligned}
$$

Furthermore, $\operatorname{since} \operatorname{sign}\left(f\left(x_{1}\right)\right)=\operatorname{sign}\left(f\left(x_{2}\right)\right)=-1$ but $\eta\left(x_{1}\right), \eta\left(x_{2}\right) \geq 1 / 2$,

$$
\begin{aligned}
R(f)-R^{*} & =\mathbf{E}|2 \eta(X)-1| \\
& =\gamma\left(2 \eta\left(x_{1}\right)-1\right)+(1-\gamma)\left(2 \eta\left(x_{2}\right)-1\right) \\
& =\theta
\end{aligned}
$$

For Part 3, first note that, for any $\phi, \psi$ is continuous on $[0,1]$ and $\psi(0)=0$ by Lemma 5 , parts 6,8 , and hence $\theta_{i} \rightarrow 0$ implies $\psi\left(\theta_{i}\right) \rightarrow 0$. Thus, we can replace condition (3b) by

$(3 \mathrm{~b})$ For any sequence $\left(\theta_{i}\right)$ in $[0,1]$,

$$
\psi\left(\theta_{i}\right) \rightarrow 0 \quad \text { implies } \quad \theta_{i} \rightarrow 0
$$

To see that (3a) implies (3b'), let $\phi$ be classification-calibrated, and let $\left(\theta_{i}\right)$ be a sequence that does not converge to 0 . Define $c=\lim \sup \theta_{i}>0$, and pass to a subsequence with $\lim \theta_{i}=c$. Then $\lim \psi\left(\theta_{i}\right)=\psi(c)$ by continuity, and $\psi(c)>0$ by classification-calibration (Lemma 5 , part 9 ). Thus, for the original sequence $\left(\theta_{i}\right)$, we see $\lim \sup \psi\left(\theta_{i}\right)>0$, so we cannot have $\psi\left(\theta_{i}\right) \rightarrow 0$.

To see that (3b') implies (3c), suppose that $R_{\phi}\left(f_{i}\right) \rightarrow R_{\phi}^{*}$. By Part $1, \psi\left(R\left(f_{i}\right)-R^{*}\right) \rightarrow 0$, and $\left(3 \mathrm{~b}\right.$ ') implies $R\left(f_{i}\right) \rightarrow R^{*}$.

Finally, to see that (3c) implies (3a), suppose that $\phi$ is not classification-calibrated and fix some $\eta \neq 1 / 2$. We can find a sequence $\alpha_{1}, \alpha_{2}, \ldots$ such that $\left(\alpha_{i}\right)$ achieves $H$ at $\eta$ but has $\liminf _{i \rightarrow \infty} \operatorname{sign}\left(\alpha_{i}(\eta-1 / 2)\right) \neq 1$. Replace the sequence with a subsequence that also achieves $H$ at $\eta$ but has $\lim \operatorname{sign}\left(\alpha_{i}(\eta-1 / 2)\right)=-1$. Fix $x \in \mathcal{X}$ and choose the probability distribution $P$ so that $P_{X}\{x\}=1$ and $P(Y=1 \mid X=x)=\eta$. Define a sequence of functions $f_{i}: \mathcal{X} \rightarrow \mathbb{R}$ for which $f_{i}(x)=\alpha_{i}$. Then $\lim R\left(f_{i}\right)>R^{*}$, and this is true for any infinite subsequence. But since $\alpha_{i}$ achieves $H$ at $\eta, \lim R_{\phi}\left(f_{i}\right)=R_{\phi}^{*}$.

\section{Further analysis of conditions on $\phi$}

In this section we consider additional conditions on the loss function $\phi$. In particular, we study the role of convexity. 


\subsection{Convex loss functions}

For convex $\phi$, classification-calibration is equivalent to a condition on the derivative of $\phi$ at zero. Recall that a subgradient of $\phi$ at $\alpha \in \mathbb{R}$ is any value $m_{\alpha} \in \mathbb{R}$ such that $\phi(x) \geq \phi(\alpha)+m_{\alpha}(x-\alpha)$ for all $x$.

Theorem 6. Let $\phi$ be convex. Then $\phi$ is classification-calibrated if and only if it is differentiable at 0 and $\phi^{\prime}(0)<0$.

Proof. Fix a convex function $\phi$.

$(\Longrightarrow)$ Since $\phi$ is convex, we can find subgradients $g_{1} \geq g_{2}$ such that, for all $\alpha$,

$$
\begin{aligned}
& \phi(\alpha) \geq g_{1} \alpha+\phi(0) \\
& \phi(\alpha) \geq g_{2} \alpha+\phi(0) .
\end{aligned}
$$

Then we have

$$
\begin{aligned}
\eta \phi(\alpha)+(1-\eta) \phi(-\alpha) & \geq \eta\left(g_{1} \alpha+\phi(0)\right)+(1-\eta)\left(-g_{2} \alpha+\phi(0)\right) \\
& =\left(\eta g_{1}-(1-\eta) g_{2}\right) \alpha+\phi(0) \\
& =\left(\frac{1}{2}\left(g_{1}-g_{2}\right)+\left(g_{1}+g_{2}\right)\left(\eta-\frac{1}{2}\right)\right) \alpha+\phi(0) .
\end{aligned}
$$

Since $\phi$ is classification-calibrated, for $\eta>1 / 2$ we can express $H(\eta)$ as $\inf _{\alpha>0} \eta \phi(\alpha)+(1-\eta) \phi(-\alpha)$. If (7) were greater than $\phi(0)$ for every $\alpha>0$, it would then follow that for $\eta>1 / 2, H(\eta) \geq \phi(0) \geq$ $H(1 / 2)$, which, by Lemma 5 , part 3 , is a contradiction. We now show that $g_{1}>g_{2}$ implies this contradiction. Indeed, we can choose

$$
\frac{1}{2}<\eta<\frac{1}{2}+\frac{g_{1}-g_{2}}{2\left|g_{1}+g_{2}\right|}
$$

to show that $\left|(\eta-1 / 2)\left(g_{1}+g_{2}\right)\right|<\left(g_{1}-g_{2}\right) / 2$, so (7) is greater than $\phi(0)$ for all $\alpha>0$. Thus, if $\phi$ is classification-calibrated, we must have $g_{1}=g_{2}$, which implies $\phi$ is differentiable at 0 .

To see that we must also have $\phi^{\prime}(0)<0$, notice that, from (6), we have

$$
\eta \phi(\alpha)+(1-\eta) \phi(-\alpha) \geq(2 \eta-1) \phi^{\prime}(0) \alpha+\phi(0) .
$$

But for any $\eta>1 / 2$ and $\alpha>0$, if $\phi^{\prime}(0) \geq 0$, this expression is at least $\phi(0)$. Thus, if $\phi$ is classification-calibrated, we must have $\phi^{\prime}(0)<0$.

$(\Longleftarrow)$ Suppose that $\phi$ is differentiable at 0 and has $\phi^{\prime}(0)<0$. Then the function $C_{\eta}(\alpha)=$ $\eta \phi(\alpha)+(1-\eta) \phi(-\alpha)$ has $C_{\eta}^{\prime}(0)=(2 \eta-1) \phi^{\prime}(0)$. For $\eta>1 / 2$, this is negative. It follows from the convexity of $\phi$ that $C_{\eta}(\alpha)$ is minimized by some $\alpha^{*} \in(0, \infty]$. To see this, notice that for some $\alpha_{0}>0$, we have

$$
C_{\eta}\left(\alpha_{0}\right) \leq C_{\eta}(0)+\alpha_{0} C_{\eta}^{\prime}(0) / 2 .
$$

But the convexity of $\phi$, and hence of $C_{\eta}$, implies that for all $\alpha$,

$$
C_{\eta}(\alpha) \geq C_{\eta}(0)+\alpha C_{\eta}^{\prime}(0) .
$$

In particular, if $\alpha \leq \alpha_{0} / 4$,

$$
C_{\eta}(\alpha) \geq C_{\eta}(0)+\frac{\alpha_{0}}{4} C_{\eta}^{\prime}(0)>C_{\eta}(0)+\frac{\alpha_{0}}{2} C_{\eta}^{\prime}(0) \geq C_{\eta}\left(\alpha_{0}\right)
$$

Similarly, for $\eta<1 / 2$, the optimal $\alpha$ is negative. This means that $\phi$ is classification-calibrated. 
The next lemma shows that for convex $\phi$, the $\psi$ transform is a little easier to compute.

Lemma 7. If $\phi$ is convex and classification-calibrated, then $\tilde{\psi}$ is convex, hence $\psi=\tilde{\psi}$.

Proof. Theorem 6 tells us $\phi$ is differentiable at zero and $\phi^{\prime}(0)<0$. Hence we have

$$
\begin{aligned}
\phi(0) & \geq H^{-}(\eta) \\
& =\inf _{\alpha: \alpha(\eta-1 / 2) \leq 0}(\eta \phi(\alpha)+(1-\eta) \phi(-\alpha)) \\
& \geq \inf _{\alpha: \alpha(\eta-1 / 2) \leq 0}\left(\eta\left(\phi(0)+\phi^{\prime}(0) \alpha\right)+(1-\eta)\left(\phi(0)-\phi^{\prime}(0) \alpha\right)\right) \\
& =\phi(0)+\inf _{\alpha: \alpha(\eta-1 / 2) \leq 0}\left((2 \eta-1) \phi^{\prime}(0) \alpha\right) \\
& =\phi(0) .
\end{aligned}
$$

Thus, $H^{-}(\eta)=\phi(0)$. The concavity of $H$ (Lemma 5, part 2) implies $\tilde{\psi}=H^{-}(\eta)-H(\eta)$ is convex, which gives the result.

If $\phi$ is convex and classification-calibrated, then it is differentiable at zero, and we can define the Bregman divergence of $\phi$ at 0 :

$$
d_{\phi}(0, \alpha)=\phi(\alpha)-\left(\phi(0)+\alpha \phi^{\prime}(0)\right)
$$

We consider a symmetrized, normalized version of the Bregman divergence at 0 , for $\alpha \geq 0$ :

$$
\xi(\alpha)=\frac{d_{\phi}(0, \alpha)+d_{\phi}(0,-\alpha)}{-\phi^{\prime}(0) \alpha}
$$

Since $\phi$ is convex on $\mathbb{R}$, both $\phi$ and $\xi$ are continuous, so we can define

$$
\xi^{-1}(\theta)=\inf \{\alpha: \xi(\alpha)=\theta\}
$$

Lemma 8. For convex, classification-calibrated $\phi$,

$$
\psi(\theta) \geq-\phi^{\prime}(0) \frac{\theta}{2} \xi^{-1}\left(\frac{\theta}{2}\right)
$$


Proof. From convexity of $\phi$, we have

$$
\begin{aligned}
\psi(\theta)= & H\left(\frac{1}{2}\right)-H\left(\frac{1+\theta}{2}\right) \\
= & \phi(0)-\inf _{\alpha>0}\left(\frac{1+\theta}{2} \phi(\alpha)+\frac{1-\theta}{2} \phi(-\alpha)\right) \\
= & \sup _{\alpha>0}\left(-\theta \phi^{\prime}(0) \alpha+\frac{1+\theta}{2}\left(\phi(0)-\phi(\alpha)+\alpha \phi^{\prime}(0)\right)\right. \\
& \left.\quad+\frac{1-\theta}{2}\left(\phi(0)-\phi(-\alpha)-\alpha \phi^{\prime}(0)\right)\right) \\
= & \sup _{\alpha>0}\left(-\theta \phi^{\prime}(0) \alpha-\frac{1+\theta}{2} d_{\phi}(0, \alpha)-\frac{1-\theta}{2} d_{\phi}(0,-\alpha)\right) \\
\geq & \sup _{\alpha>0}\left(-\theta \phi^{\prime}(0) \alpha-d_{\phi}(0, \alpha)-d_{\phi}(0,-\alpha)\right) \\
= & \sup _{\alpha>0}(\theta-\xi(\alpha))\left(-\phi^{\prime}(0) \alpha\right) \\
\geq & \left(\theta-\xi\left(\xi^{-1}(\theta / 2)\right)\right)\left(-\phi^{\prime}(0) \xi^{-1}(\theta / 2)\right) \\
= & -\phi^{\prime}(0) \frac{\theta}{2} \xi^{-1}\left(\frac{\theta}{2}\right) .
\end{aligned}
$$

Notice that a slower increase of $\xi$ (that is, a less curved $\phi$ ) gives better bounds on $R(f)-R^{*}$ in terms of $R_{\phi}(f)-R_{\phi}^{*}$.

\subsection{General loss functions}

All of the classification procedures mentioned in earlier sections utilize surrogate loss functions which are either upper bounds on 0-1 loss or can be transformed into upper bounds via a positive scaling factor. This is not a coincidence: as the next lemma establishes, it must be possible to scale any classification-calibrated $\phi$ into such a majorant.

Lemma 9. If $\phi: \mathbb{R} \rightarrow[0, \infty)$ is classification-calibrated, then there is a $\gamma>0$ such that $\gamma \phi(\alpha) \geq$ $\mathbf{1}[\alpha \leq 0]$ for all $\alpha \in \mathbb{R}$.

Proof. Proceeding by contrapositive, suppose no such $\gamma$ exists. Since $\phi(\alpha) \geq \mathbf{1}[\alpha \leq 0]$ on $(0, \infty)$, we must then have $\inf _{\alpha \leq 0} \phi(\alpha)=0$. But $\phi(\alpha)=C_{1}(\alpha)$, hence

$$
0=\inf _{\alpha \leq 0} C_{1}(\alpha)=H^{-}(1) \geq H(1) \geq 0 .
$$

Thus, $H^{-}(1)=H(1)$, so $\phi$ is not classification-calibrated.

We have seen that for convex $\phi$, the function $\tilde{\psi}$ is convex, and so $\psi=\tilde{\psi}$. The following example shows that we cannot, in general, avoid computing the convex lower bound $\psi$. 
Example 5. Consider the following (classification-calibrated) loss function; see the left panel of Figure 6.

$$
\phi(\alpha)= \begin{cases}4 & \text { if } \alpha \leq 0, \alpha \neq-1 \\ 3 & \text { if } \alpha=-1 \\ 2 & \text { if } \alpha=1 \\ 0 & \text { if } \alpha>0, \alpha \neq 1\end{cases}
$$

Then $\tilde{\psi}$ is not convex, so $\psi \neq \tilde{\psi}$.

Proof. It is easy to check that

$$
H^{-}(\eta)= \begin{cases}\min \{4 \eta, 2+\eta\} & \text { if } \eta \geq 1 / 2 \\ \min \{4(1-\eta), 3-\eta\} & \text { if } \eta<1 / 2\end{cases}
$$

and that $H(\eta)=4 \min \{\eta, 1-\eta\}$. Thus,

so

$$
H^{-}(\eta)-H(\eta)= \begin{cases}\min \{8 \eta-4,5 \eta-2\} & \text { if } \eta \geq 1 / 2 \\ \min \{4-8 \eta, 3-5 \eta\} & \text { if } \eta<1 / 2\end{cases}
$$

$$
\tilde{\psi}(\theta)=\min \left\{4 \theta, \frac{1}{2}(5 \theta+1)\right\} .
$$

This function, illustrated in the right panel of Figure 6, is not convex; in fact it is concave.

\section{Tighter bounds under low noise conditions}

In a study of the convergence rate of empirical risk minimization, Tsybakov (2001) provided a useful condition on the behavior of the posterior probability near the optimal decision boundary $\{x: \eta(x)=1 / 2\}$. Tsybakov's condition is useful in our setting as well; as we show in this section, it allows us to obtain a refinement of Theorem 3 .

Recall that

$$
\begin{aligned}
R(f)-R^{*} & =\mathbf{E}(\mathbf{1}[\operatorname{sign}(f(X)) \neq \operatorname{sign}(\eta(X)-1 / 2)]|2 \eta(X)-1|) \\
& \leq P_{X}(\operatorname{sign}(f(X)) \neq \operatorname{sign}(\eta(X)-1 / 2)),
\end{aligned}
$$

with equality provided that $\eta(X)$ is almost surely either 1 or 0 . We say that $P$ has noise exponent $\alpha \geq 0$ if there is a $c>0$ such that every measurable $f: \mathcal{X} \rightarrow \mathbb{R}$ has

$$
P_{X}(\operatorname{sign}(f(X)) \neq \operatorname{sign}(\eta(X)-1 / 2)) \leq c\left(R(f)-R^{*}\right)^{\alpha} .
$$

Notice that we must have $\alpha \leq 1$, in view of (8). If $\alpha=0$, this imposes no constraint on the noise: take $c=1$ to see that every probability measure $P$ satisfies (9). On the other hand, $\alpha=1$ if and only if $|2 \eta(X)-1| \geq 1 / c$ a.s. $\left[P_{X}\right]$. The reverse implication is immediate; to see the forward implication, notice that the condition must apply for every measurable $f$. For $\alpha=1$ it requires that

$$
\begin{aligned}
(\forall A \in \mathcal{G}) P(A) & \leq c \int_{A}|2 \eta(X)-1| d P_{X} \\
\Longleftrightarrow(\forall A \in \mathcal{G}) \int_{A} \frac{1}{c} d P_{X} & \leq \int_{A}|2 \eta(X)-1| d P_{X} \\
\Longleftrightarrow \frac{1}{c} & \leq|2 \eta(X)-1| \text { a.s. }\left[P_{X}\right] .
\end{aligned}
$$



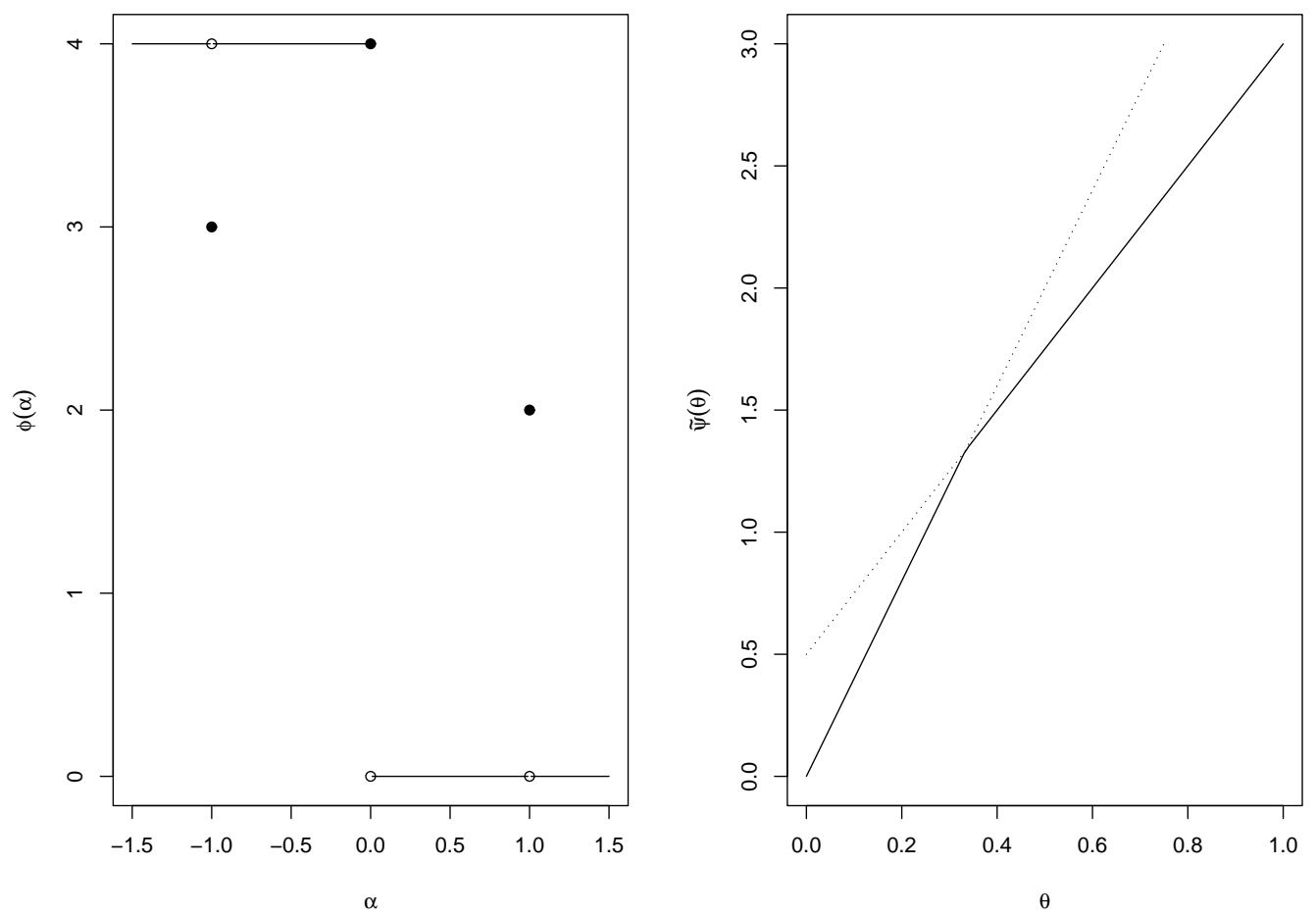

Figure 6: Left panel, the loss function of Example 5. Right panel, the corresponding (nonconvex) $\tilde{\psi}$. The dotted lines depict the graphs for the two linear functions of which $\tilde{\psi}$ is a pointwise minimum.

Theorem 10. Suppose $P$ has noise exponent $0<\alpha \leq 1$, and $\phi$ is classification-calibrated and error-averse. Then there is a $c>0$ such that for any $f: \mathcal{X} \rightarrow \mathbb{R}$,

$$
c\left(R(f)-R^{*}\right)^{\alpha} \psi\left(\frac{\left(R(f)-R^{*}\right)^{1-\alpha}}{2 c}\right) \leq R_{\phi}(f)-R_{\phi}^{*} .
$$

Furthermore, this never gives a worse rate than the result of Theorem 3, since

$$
\left(R(f)-R^{*}\right)^{\alpha} \psi\left(\frac{\left(R(f)-R^{*}\right)^{1-\alpha}}{2 c}\right) \geq \psi\left(\frac{R(f)-R^{*}}{2 c}\right)
$$

Proof. Fix $c>0$ such that for every $f: \mathcal{X} \rightarrow \mathbb{R}$,

$$
P_{X}(\operatorname{sign}(f(X)) \neq \operatorname{sign}(\eta(X)-1 / 2)) \leq c\left(R(f)-R^{*}\right)^{\alpha} .
$$

We approximate the error integral separately over a region with high noise, and over the remainder 
of the input space. To this end, fix $\epsilon>0$ (the noise threshold), and notice that

$$
\begin{aligned}
& R(f)-R^{*}=\mathbf{E}(\mathbf{1}[\operatorname{sign}(f(X)) \neq \operatorname{sign}(\eta(X)-1 / 2)]|2 \eta(X)-1|) \\
&=\mathbf{E}(\mathbf{1}[|2 \eta(X)-1|<\epsilon] \mathbf{1}[\operatorname{sign}(f(X)) \neq \operatorname{sign}(\eta(X)-1 / 2)]|2 \eta(X)-1|) \\
& \quad+\mathbf{E}(\mathbf{1}[|2 \eta(X)-1| \geq \epsilon] \mathbf{1}[\operatorname{sign}(f(X)) \neq \operatorname{sign}(\eta(X)-1 / 2)]|2 \eta(X)-1|) \\
& \leq c \epsilon\left(R(f)-R^{*}\right)^{\alpha} \\
& \quad+\mathbf{E}(\mathbf{1}[|2 \eta(X)-1| \geq \epsilon] \mathbf{1}[\operatorname{sign}(f(X)) \neq \operatorname{sign}(\eta(X)-1 / 2)]|2 \eta(X)-1|) .
\end{aligned}
$$

Now, for any $x$,

$$
\mathbf{1}[|2 \eta(x)-1| \geq \epsilon]|2 \eta(x)-1| \leq \frac{\epsilon}{\psi(\epsilon)} \psi(|2 \eta(x)-1|) .
$$

Indeed, when $|2 \eta(x)-1|<\epsilon$, (10) follows from the fact that $\psi$ is nonnegative (Lemma 5, parts $8,9)$, and when $|2 \eta(x)-1| \geq \epsilon$ it follows from Lemma $4(2)$.

Thus, using the same argument as in the proof of Theorem 3 ,

$$
\begin{aligned}
R(f)-R^{*} & \leq c \epsilon\left(R(f)-R^{*}\right)^{\alpha}+\frac{\epsilon}{\psi(\epsilon)} \mathbf{E}(\mathbf{1}[\operatorname{sign}(f(X)) \neq \operatorname{sign}(\eta(X)-1 / 2)] \psi(|2 \eta(X)-1|)) \\
& \leq c \epsilon\left(R(f)-R^{*}\right)^{\alpha}+\frac{\epsilon}{\psi(\epsilon)}\left(R_{\phi}(f)-R_{\phi}^{*}\right)
\end{aligned}
$$

and hence,

$$
\left(\frac{R(f)-R^{*}}{\epsilon}-c\left(R(f)-R^{*}\right)^{\alpha}\right) \psi(\epsilon) \leq R_{\phi}(f)-R_{\phi}^{*}
$$

Choosing

$$
\epsilon=\frac{1}{2 c}\left(R(f)-R^{*}\right)^{1-\alpha}
$$

and substituting gives the first inequality. (We can assume that $R(f)-R^{*}>0$, since the inequality is trivial otherwise.)

The second inequality follows from the fact that $\psi(\theta) / \theta$ is non-decreasing, which we know from Lemma 4, part 3.

\section{Estimation rates}

In previous sections, we have seen that the excess risk, $R(f)-R^{*}$, can be bounded in terms of the excess $\phi$-risk, $R_{\phi}(f)-R_{\phi}^{*}$. Many large margin algorithms choose $\hat{f}$ to minimize the empirical $\phi$-risk,

$$
\hat{R}_{\phi}(f)=\hat{\mathbf{E}} \phi(Y f(X))=\frac{1}{n} \sum_{i=1}^{n} \phi\left(Y_{i} f\left(X_{i}\right)\right) .
$$

In this section, we examine the convergence of $\hat{f}$ 's excess $\phi$-risk, $R_{\phi}(\hat{f})-R_{\phi}^{*}$. We can split this excess risk into an estimation error term and an approximation error term:

$$
R_{\phi}(\hat{f})-R_{\phi}^{*}=\left(R_{\phi}(\hat{f})-\inf _{f \in \mathcal{F}} R_{\phi}(f)\right)+\left(\inf _{f \in \mathcal{F}} R_{\phi}(f)-R_{\phi}^{*}\right)
$$


We focus on the first term, the estimation error term. We assume throughout that some $f^{*} \in \mathcal{F}$ achieves the infimum,

$$
R_{\phi}\left(f^{*}\right)=\inf _{f \in \mathcal{F}} R_{\phi}(f) .
$$

The simplest way to bound $R_{\phi}(\hat{f})-R_{\phi}\left(f^{*}\right)$ is to use a uniform convergence argument: if

$$
\sup _{f \in \mathcal{F}}\left|\hat{R}_{\phi}(f)-R_{\phi}(f)\right| \leq \epsilon_{n},
$$

then

$$
\begin{aligned}
R_{\phi}(\hat{f})-R_{\phi}\left(f^{*}\right) & =\left(R_{\phi}(\hat{f})-\hat{R}_{\phi}(\hat{f})\right)+\left(\hat{R}_{\phi}(\hat{f})-\hat{R}_{\phi}\left(f^{*}\right)\right)+\left(\hat{R}_{\phi}\left(f^{*}\right)-R_{\phi}\left(f^{*}\right)\right) \\
& \leq 2 \epsilon_{n}+\left(\hat{R}_{\phi}(\hat{f})-\hat{R}_{\phi}\left(f^{*}\right)\right) \\
& \leq 2 \epsilon_{n},
\end{aligned}
$$

since $\hat{f}$ minimizes $\hat{R}_{\phi}$.

This approach can give the wrong rate. For example, for a nontrivial class $\mathcal{F}$, the expectation of the empirical process in (11) can decrease no faster than $1 / \sqrt{n}$. However, if $\mathcal{F}$ is a small class (for instance, a VC-class) and $R_{\phi}\left(f^{*}\right)=0$, then $R_{\phi}(\hat{f})$ should decrease as $1 / n$.

Lee et al. (1996) showed that fast rates are also possible for the quadratic loss $\phi(\alpha)=(1-\alpha)^{2}$ if $\mathcal{F}$ is convex, even if $R_{\phi}\left(f^{*}\right)>0$. In particular, because the quadratic loss function is strictly convex, it is possible to bound the variance of the excess loss (difference between the loss of a function $f$ and that of the optimal $f^{*}$ ) in terms of its expectation. Since the variance decreases as we approach the optimal $f^{*}$, the risk of the empirical minimizer converges more quickly to the optimal risk than the simple uniform convergence results would suggest. Mendelson (2002) improved this result, and extended it from prediction in $L_{2}\left(P_{X}\right)$ to prediction in $L_{p}\left(P_{X}\right)$ for other values of $p$. The proof used the idea of the modulus of convexity of a norm. In this section, we use this idea to give a simpler proof of a more general bound when the loss function satisfies a strict convexity condition, and we obtain risk bounds. The modulus of convexity of an arbitrary strictly convex function (rather than a norm) is a key notion in formulating our results.

Definition 11 (Modulus of convexity). Given a pseudometric $d$ defined on a vector space $S$, and a convex function $f: S \rightarrow \mathbb{R}$, the modulus of convexity of $f$ with respect to $d$ is the function $\delta:[0, \infty) \rightarrow[0, \infty]$ satisfying

$$
\delta(\epsilon)=\inf \left\{\frac{f\left(x_{1}\right)+f\left(x_{2}\right)}{2}-f\left(\frac{x_{1}+x_{2}}{2}\right): x_{1}, x_{2} \in S, d\left(x_{1}, x_{2}\right) \geq \epsilon\right\} .
$$

If $\delta(\epsilon)>0$ for all $\epsilon>0$, we say that $f$ is strictly convex with respect to $d$.

We consider loss functions $\phi$ that also satisfy a Lipschitz condition with respect to a pseudometric $d$ on $\mathbb{R}$ : we say that $\phi: \mathbb{R} \rightarrow \mathbb{R}$ is Lipschitz with respect to $d$, with constant $L$, if

$$
\text { for all } a, b \in \mathbb{R},|\phi(a)-\phi(b)| \leq L \cdot d(a, b) \text {. }
$$

(Note that if $d$ is a metric and $\phi$ is convex, then $\phi$ necessarily satisfies a Lipshitz condition on any compact subset of $\mathbb{R}$ (Rockafellar, 1997).) 
In the following theorem, we use the expectation of a centered empirical process as a measure of the complexity of the class $\mathcal{F}$; define

$$
\xi_{\mathcal{F}}(\epsilon)=\mathbf{E} \sup \{\mathbf{E} f-\hat{\mathbf{E}} f: f \in \mathcal{F}, \mathbf{E} f=\epsilon\}
$$

Define the excess loss class $g_{\mathcal{F}}$ as

$$
g_{\mathcal{F}}=\left\{g_{f}: f \in \mathcal{F}\right\}=\left\{(x, y) \mapsto \phi(y f(x))-\phi\left(y f^{*}(x)\right): f \in \mathcal{F}\right\}
$$

where $f^{*}=\arg \min _{f \in \mathcal{F}} \mathbf{E} \phi(Y f(X))$.

Theorem 12. There is a constant $K$ for which the following holds. For a pseudometric $d$ on $\mathbb{R}$, suppose that $\phi: \mathbb{R} \rightarrow \mathbb{R}$ is Lipschitz with constant $L$ and convex with modulus of convexity $\delta(\epsilon) \geq c \epsilon^{r}$ (both with respect to $d$ ). Define $\beta=\min (1,2 / r)$. Fix a convex class $\mathcal{F}$ of real functions on $\mathcal{X}$ such that for all $f \in \mathcal{F}, x_{1}, x_{2} \in \mathcal{X}$, and $y_{1}, y_{2} \in \mathcal{Y}, d\left(y_{1} f\left(x_{1}\right), y_{2} f\left(x_{2}\right)\right) \leq$ B. For i.i.d. data $\left(X_{1}, Y_{1}\right), \ldots,\left(X_{n}, Y_{n}\right)$, let $\hat{f} \in \mathcal{F}$ be the minimizer of the empirical $\phi$-risk, $R_{\phi}(f)=\hat{\mathbf{E}} \phi(Y f(X))$. Then with probability at least $1-e^{-x}$,

$$
R_{\phi}(\hat{f}) \leq R_{\phi}\left(f^{*}\right)+\epsilon
$$

where

$$
\begin{aligned}
\epsilon & =K \max \left\{\epsilon^{*},\left(\frac{c_{r} L^{2} x}{n}\right)^{1 /(2-\beta)}, \frac{B L x}{n}\right\}, \\
\epsilon^{*} \geq \xi_{g_{\mathcal{F}}}\left(\epsilon^{*}\right), & \\
c_{r} & = \begin{cases}(2 c)^{-2 / r} & \text { if } r \geq 2, \\
(2 c)^{-1} B^{2-r} & \text { otherwise. }\end{cases}
\end{aligned}
$$

Thus, for any probability distribution $P$ on $\mathcal{X} \times \mathcal{Y}$ that has noise exponent $\alpha$, there is a constant $c^{\prime}$ such that, with probability at least $1-e^{-x}$,

$$
c^{\prime}\left(R(\hat{f})-R^{*}\right)^{\alpha} \psi\left(\frac{\left(R(\hat{f})-R^{*}\right)^{1-\alpha}}{2 c^{\prime}}\right) \leq \epsilon+\inf _{f \in \mathcal{F}} R_{\phi}(f)-R_{\phi}^{*} .
$$

\subsection{Proof of Theorem 12}

There are two key ingredients in the proof. Firstly, the following result shows that if the variance of an excess loss function is bounded in terms of its expectation, then we can obtain faster rates than would be implied by the uniform convergence bounds. Secondly, simple conditions on the loss function ensure that this condition is satisfied for convex function classes.

Lemma 13. Consider a class $\mathcal{F}$ of functions $f: \mathcal{X} \rightarrow \mathbb{R}$ with $\sup _{f \in \mathcal{F}}\|f\|_{\infty} \leq B$. Let $P$ be a probability distribution on $\mathcal{X}$, and suppose that there are $c \geq 1$ and $0<\beta \leq 1$ such that, for all $f \in \mathcal{F}$,

$$
\mathbf{E} f^{2}(X) \leq c(\mathbf{E} f)^{\beta}
$$


Fix $0<\alpha, \epsilon<1$. Suppose that if some $f \in \mathcal{F}$ has $\hat{\mathbf{E}} f \leq \alpha \epsilon$ and $\mathbf{E} f \geq \epsilon$, then some $f^{\prime} \in \mathcal{F}$ has $\hat{\mathbf{E}} f^{\prime} \leq \alpha \epsilon$ and $\mathbf{E} f=\epsilon$. Then with probability at least $1-e^{-x}$, any $f \in \overline{\mathcal{F}}$ satisfies

$$
\hat{\mathbf{E}} f \leq \alpha \epsilon \Rightarrow \mathbf{E} f \leq \epsilon .
$$

provided that

$$
\epsilon \geq \max \left\{\epsilon^{*},\left(\frac{9 c K x}{(1-\alpha)^{2} n}\right)^{1 /(2-\beta)}, \frac{4 K B x}{(1-\alpha) n}\right\} .
$$

where $K$ is an absolute constant and

$$
\epsilon^{*} \geq \frac{6}{1-\alpha} \xi_{\mathcal{F}}\left(\epsilon^{*}\right)
$$

As an aside, notice that Tsybakov's condition Tsybakov (2001) is of the form (12). To see this, let $f^{*}$ be the Bayes decision rule, and consider the class of functions $\left\{\alpha g_{f}: f \in \mathcal{F}, \alpha \in[0,1]\right\}$, where

$$
g_{f}(x, y)=\ell(f(x), y)-\ell\left(f^{*}(x), y\right)
$$

and $\ell$ is the discrete loss. Then the condition

$$
P_{X}\left(f(X) \neq f^{*}(X)\right) \leq c\left(\mathbf{E} \ell(f(X), Y)-\mathbf{E} \ell\left(f^{*}(X), Y\right)\right)^{\alpha}
$$

can be rewritten

$$
\mathbf{E} g_{f}^{2}(X, Y) \leq c\left(\mathbf{E} g_{f}(X, Y)\right)^{\alpha} .
$$

Thus, we can obtain a version of Tsybakov's result for small function classes from Lemma 13: if the Bayes decision rule $f^{*}$ is in $\mathcal{F}$, then the function $\hat{f}$ that minimizes empirical risk has

$$
\hat{\mathbf{E}} g_{\hat{f}}=\hat{R}(f)-\hat{R}\left(f^{*}\right) \leq 0,
$$

and so with high probability has $\mathbf{E} g_{\hat{f}}=R(f)-R^{*} \leq \epsilon$ under the conditions of the theorem. If $\mathcal{F}$ is a VC-class, we have $\epsilon \leq c \log n / n$ for some constant $c$, which is surprisingly fast when $R^{*}>0$.

The proof of Lemma 13 uses techniques from Massart (2000b), Mendelson (2002), and Bartlett et al. (2003), as well as the following concentration inequality, which is a refinement, due to Rio (2001) and Klein (2002) of a result of Massart (2000a), following Talagrand (1994), Ledoux (2001). The best estimates on the constants are due to Bousquet (2002).

Lemma 14. There is an absolute constant $K$ for which the following holds. Let $\mathcal{G}$ be a class of functions defined on $\mathcal{X}$ with $\sup _{g \in \mathcal{G}}\|g\|_{\infty} \leq b$. Suppose that $P$ is a probability distribution such that for every $g \in \mathcal{G}, \mathbf{E} g=0$. Let $X_{1}, \ldots, X_{n}$ be independent random variables distributed according to $P$ and set $\sigma^{2}=\sup _{g \in \mathcal{G}} \operatorname{var} g$. Define

$$
Z=\sup _{g \in \mathcal{G}} \frac{1}{n} \sum_{i=1}^{n} g\left(X_{i}\right) .
$$

Then, for every $x>0$ and every $\rho>0$,

$$
\operatorname{Pr}\left\{Z \geq(1+\rho) \mathbf{E} Z+\sigma \sqrt{\frac{K x}{n}}+\frac{K\left(1+\rho^{-1}\right) b x}{n}\right\} \leq e^{-x} .
$$


Proof. (of Lemma 13)

From the condition on $\mathcal{F}$, we have

$$
\begin{aligned}
\operatorname{Pr}\{\exists f \in \mathcal{F}: \hat{\mathbf{E}} f \leq \alpha \epsilon, \mathbf{E} f \geq \epsilon\} & \leq \operatorname{Pr}\{\exists f \in \mathcal{F}: \hat{\mathbf{E}} f \leq \alpha \epsilon, \mathbf{E} f=\epsilon\} \\
& =\operatorname{Pr}\{\sup \{\mathbf{E} f-\hat{\mathbf{E}} f: f \in \mathcal{F}, \mathbf{E} f=\epsilon\} \geq(1-\alpha) \epsilon\}
\end{aligned}
$$

We bound this probability using Lemma 14, with $\rho=1$ and $\mathcal{G}=\{\mathbf{E} f-f: f \in \mathcal{F}, \mathbf{E} f=\epsilon\}$. This shows that

$$
\operatorname{Pr}\{\exists f \in \mathcal{F}: \hat{\mathbf{E}} f \leq \alpha \epsilon, \mathbf{E} f \geq \epsilon\} \leq \operatorname{Pr}\{Z \geq(1-\alpha) \epsilon\} \leq e^{-x}
$$

provided that

$$
\begin{aligned}
2 \mathbf{E} Z & \leq \frac{(1-\alpha) \epsilon}{3}, \\
\sqrt{\frac{c \epsilon^{\beta} K x}{n}} & \leq \frac{(1-\alpha) \epsilon}{3}, \text { and } \\
\frac{4 K B x}{n} & \leq \frac{(1-\alpha) \epsilon}{3} .
\end{aligned}
$$

(We have used the fact that $\sup _{f \in \mathcal{F}}\|f\|_{\infty} \leq B$ implies $\sup _{g \in \mathcal{G}}\|g\|_{\infty} \leq 2 B$.) Observing that

$$
\mathbf{E} Z=\xi_{\mathcal{F}}(\epsilon)
$$

and rearranging gives the result.

The second ingredient in the proof of Theorem 12 is the following lemma, which gives conditions that ensure a variance bound of the kind required for the previous lemma (condition (12)). For a pseudometric $d$ on $\mathbb{R}$ and a probability distribution on $\mathcal{X}$, we can define a pseudometric $\tilde{d}$ on the set of uniformly bounded real functions on $\mathcal{X}$,

$$
\tilde{d}(f, g)=\left(\mathbf{E} d(f(X), g(X))^{2}\right)^{1 / 2} .
$$

If $d$ is the usual metric on $\mathbb{R}$, then $\tilde{d}$ is the $L_{2}(P)$ pseudometric.

Lemma 15. Consider a convex class $\mathcal{F}$ of real-valued functions defined on $\mathcal{X}$, a convex loss function $\ell: \mathbb{R} \rightarrow \mathbb{R}$, and a pseudometric $d$ on $\mathbb{R}$. Suppose that $\ell$ satisfies the following conditions.

1. $\ell$ is Lipschitz with respect to $d$, with constant $L$ :

$$
\text { for all } a, b \in \mathbb{R},|\ell(a)-\ell(b)| \leq L d(a, b) \text {. }
$$

2. $R(f)=\mathbf{E} \ell(f)$ is a strictly convex functional with respect to the pseudometric $\tilde{d}$, with modulus of convexity $\tilde{\delta}$ :

$$
\tilde{\delta}(\epsilon)=\inf \left\{\frac{R(f)+R(g)}{2}-R\left(\frac{f+g}{2}\right): \tilde{d}(f, g) \geq \epsilon\right\}
$$


Suppose that $f^{*}$ satisfies $R\left(f^{*}\right)=\inf _{f \in \mathcal{F}} R(f)$, and define

$$
g_{f}(x)=\ell(f(x))-\ell\left(f^{*}(x)\right) .
$$

Then

$$
\mathbf{E} g_{f} \geq 2 \tilde{\delta}\left(\tilde{d}\left(f, f^{*}\right)\right) \geq 2 \tilde{\delta}\left(\frac{\sqrt{\mathbf{E} g_{f}^{2}}}{L}\right)
$$

We shall apply the lemma to a class of functions of the form $(x, y) \mapsto y f(x)$, with the loss function $\ell=\phi$. (The lemma can be trivially extended to a loss function $\ell: \mathbb{R} \times \mathcal{Y} \rightarrow \mathbb{R}$ that satisfies a Lipschitz constraint uniformly over $\mathcal{Y}$.)

Proof. The proof proceeds in two steps: the Lipschitz condition allows us to relate $\mathbf{E} g_{f}^{2}$ to $\tilde{d}\left(f, f^{*}\right)$, and the modulus of convexity condition, together with the convexity of $\mathcal{F}$, relates this to $\mathbf{E} g_{f}$.

We have

$$
\begin{aligned}
\mathbf{E} g_{f}^{2} & =\mathbf{E}\left(\ell(f(X))-\ell\left(f^{*}(X)\right)\right)^{2} \\
& \leq \mathbf{E}\left(L d\left(f(X), f^{*}(X)\right)\right)^{2} \\
& =L^{2}\left(\tilde{d}\left(f, f^{*}\right)\right)^{2} .
\end{aligned}
$$

From the definition of the modulus of convexity,

$$
\begin{aligned}
\frac{R(f)+R\left(f^{*}\right)}{2} & \geq R\left(\frac{f+f^{*}}{2}\right)+\tilde{\delta}\left(\tilde{d}\left(f, f^{*}\right)\right) \\
& \geq R\left(f^{*}\right)+\tilde{\delta}\left(\tilde{d}\left(f, f^{*}\right)\right),
\end{aligned}
$$

where the optimality of $f^{*}$ in the convex set $\mathcal{F}$ implies the second inequality. Rearranging gives

$$
\mathbf{E} g_{f}=R(f)-R\left(f^{*}\right) \geq 2 \tilde{\delta}\left(\tilde{d}\left(f, f^{*}\right)\right) .
$$

Combining with (13) gives the result.

In our application, the following result will imply that we can estimate the modulus of convexity of $R_{\phi}$ with respect to the pseudometric $\tilde{d}$ if we have some information about the modulus of convexity of $\phi$ with respect to the pseudometric $d$.

Lemma 16. Suppose that a convex function $\ell: \mathbb{R} \rightarrow \mathbb{R}$ has modulus of convexity $\delta$ with respect to a pseudometric $d$ on $\mathbb{R}$, for some fixed $c, r>0$, every $\epsilon>0$ satisfies

$$
\delta(\epsilon) \geq c \epsilon^{r} .
$$

Then for functions $f: \mathcal{X} \rightarrow \mathbb{R}$ satisfying $\sup _{x_{1}, x_{2}} d\left(f\left(x_{1}\right), f\left(x_{2}\right)\right)=B$, the modulus of convexity $\tilde{\delta}$ of $R(f)=\mathbf{E} \ell(f)$ with respect to the pseudometric $\tilde{d}$ satisfies

$$
\tilde{\delta}(\epsilon) \geq c_{r} \epsilon^{\max \{2, r\}},
$$

where $c_{r}=c$ if $r \geq 2$ and $c_{r}=c B^{r-2}$ otherwise. 
Proof. Fix functions $f_{1}, f_{2}: \mathcal{X} \rightarrow \mathbb{R}$ with $\tilde{d}\left(f_{1}, f_{2}\right)=\sqrt{\mathbf{E} d^{2}\left(f_{1}(X), f_{2}(X)\right)} \geq \epsilon$. We have

$$
\begin{aligned}
\frac{R\left(f_{1}\right)+R\left(f_{2}\right)}{2}-R\left(\frac{f_{1}+f_{2}}{2}\right) & =\mathbf{E}\left(\frac{\ell\left(f_{1}(X)\right)+\ell\left(f_{2}(X)\right)}{2}-\ell\left(\frac{f_{1}(X)+f_{2}(X)}{2}\right)\right) \\
& \geq \mathbf{E}\left(\delta\left(d\left(f_{1}(X), f_{2}(X)\right)\right)\right) \\
& \geq c \mathbf{E} d^{r}\left(f_{1}(X), f_{2}(X)\right) \\
& =c \mathbf{E}\left(d^{2}\left(f_{1}(X), f_{2}(X)\right)\right)^{r / 2} .
\end{aligned}
$$

When the function $\xi(a)=a^{r / 2}$ is convex (i.e., when $r \geq 2$ ), Jensen's inequality shows that

$$
\frac{R\left(f_{1}\right)+R\left(f_{2}\right)}{2}-R\left(\frac{f_{1}+f_{2}}{2}\right) \geq c \epsilon^{r} \text {. }
$$

Otherwise, we use the following convex lower bound on $\xi:\left[0, B^{2}\right] \rightarrow\left[0, B^{r}\right]$,

$$
\xi(a)=a^{r / 2} \geq B^{r} \frac{a}{B^{2}},
$$

which follows from (the concave analog of) Lemma 4, part 2. This implies

$$
\frac{R\left(f_{1}\right)+R\left(f_{2}\right)}{2}-R\left(\frac{f_{1}+f_{2}}{2}\right) \geq c B^{r-2} \epsilon^{2} .
$$

It is also possible to prove a converse result, that the modulus of convexity of $\phi$ is at least the infimum over probability distributions of the modulus of convexity of $R$. (To see this, we choose a probability distribution concentrated on the $x \in \mathcal{X}$ where $f_{1}(x)$ and $f_{2}(x)$ achieve the infimum in the definition of the modulus of convexity.)

Proof. (of Theorem 12) Consider the class $\left\{g_{f}: f \in \mathcal{F}\right\}$ with, for each $f \in \mathcal{F}$,

$$
g_{f}(x, y)=\phi(y f(x))-\phi\left(y f^{*}(x)\right)
$$

where $f^{*} \in \mathcal{F}$ minimizes $R_{\phi}(f)=\mathbf{E} \phi(Y f(X))$. Applying Lemma 16, we see that the functional $R(f)=\mathbf{E} \phi(f)$, defined for functions $(x, y) \mapsto y f(x)$, has modulus of convexity

$$
\tilde{\delta}(\epsilon) \geq c_{r} \epsilon^{\max \{2, r\}},
$$

where $c_{r}=c$ if $r \geq 2$ and $c_{r}=c B^{r-2}$ otherwise. From Lemma 15,

$$
\mathbf{E} g_{f} \geq 2 c_{r}\left(\frac{\sqrt{\mathbf{E} g_{f}^{2}}}{L}\right)^{\max \{2, r\}},
$$

which is equivalent to

$$
\mathbf{E} g_{f}^{2} \leq c_{r}^{\prime} L^{2}\left(\mathbf{E} g_{f}\right)^{\min \{1,2 / r\}}
$$

with

$$
c_{r}^{\prime}= \begin{cases}(2 c)^{-2 / r} & \text { if } r \geq 2 \\ (2 c)^{-1} B^{2-r} & \text { otherwise }\end{cases}
$$


To apply Lemma 13 to the class $\left\{g_{f}: f \in \mathcal{F}\right\}$, we need to check the condition. Suppose that $g_{f}$ has $\hat{\mathbf{E}} g_{f} \leq \alpha \epsilon$ and $\mathbf{E} g_{f} \geq \epsilon$. Then, by the convexity of $\mathcal{F}$ and the continuity of $\phi$, some $f^{\prime}=\gamma f+(1-\gamma) f^{*} \in \mathcal{F}$, for $0 \leq \gamma \leq 1$, has $\mathbf{E} g_{f}=\epsilon$. Jensen's inequality shows that

$$
\hat{\mathbf{E}} g_{f}=\hat{\mathbf{E}} \phi\left(Y\left(\gamma f(X)+(1-\gamma) f^{*}(X)\right)\right)-\hat{\mathbf{E}} \phi\left(Y f^{*}(X)\right) \leq \gamma\left(\hat{\mathbf{E}} \phi(Y f(x))-\hat{\mathbf{E}} \phi\left(Y f^{*}(X)\right)\right) \leq \alpha \epsilon .
$$

Applying Lemma 13 we have, with probability at least $1-e^{-x}$, any $g_{f}$ with $\hat{\mathbf{E}} g_{f} \leq \epsilon / 2$ also has $\mathbf{E} g_{f} \leq \epsilon$, provided

$$
\epsilon \geq \max \left\{\epsilon^{*},\left(\frac{36 c_{r}^{\prime} L^{2} K x}{n}\right)^{1 /(2-\min \{1,2 / r\})}, \frac{16 K B L x}{n}\right\},
$$

where $\epsilon^{*} \geq 12 \xi_{g_{\mathcal{F}}}\left(\epsilon^{*}\right)$. In particular, if $\hat{f} \in \mathcal{F}$ minimizes empirical risk, then

$$
\hat{\mathbf{E}} g_{\hat{f}}=\hat{R}_{\phi}(\hat{f})-\hat{R}_{\phi}\left(f^{*}\right) \leq 0<\frac{\epsilon}{2}
$$

hence $\mathbf{E} g_{\hat{f}} \leq \epsilon$.

Combining with Theorem 10 shows that, for some $c^{\prime}$,

$$
\begin{aligned}
c^{\prime}\left(R(\hat{f})-R^{*}\right)^{\alpha} \psi\left(\frac{\left(R(\hat{f})-R^{*}\right)^{1-\alpha}}{2 c^{\prime}}\right) & \leq R_{\phi}(\hat{f})-R_{\phi}^{*} \\
& =R_{\phi}(\hat{f})-R_{\phi}\left(f^{*}\right)+R_{\phi}\left(f^{*}\right)-R_{\phi}^{*} \\
& \leq \epsilon+R_{\phi}\left(f^{*}\right)-R_{\phi}^{*} .
\end{aligned}
$$

\subsection{Examples}

We consider four loss functions that satisfy the requirements for the fast convergence rates: the exponential loss function used in AdaBoost, the deviance function corresponding to logistic regression, the quadratic loss function, and the truncated quadratic loss function; see Table 1 . These functions are illustrated in Figures 1 and 3. We use the pseudometric

$$
d_{\phi}(a, b)=\inf \{|a-\alpha|+|\beta-b|: \phi \text { constant on }(\min \{\alpha, \beta\}, \max \{\alpha, \beta\})\} .
$$

For all except the truncated quadratic loss function, this corresponds to the standard metric on $\mathbb{R}, d_{\phi}(a, b)=|a-b|$. In all cases, $d_{\phi}(a, b) \leq|a-b|$, but for the truncated quadratic, $d_{\phi}$ ignores differences to the right of 1 . It is easy to calculate the Lipschitz constant and modulus of convexity for each of these loss functions. These parameters are given in Table 1.

In the following result, we consider the function class used by algorithms such as AdaBoost: the class of linear combinations of classifiers from a fixed base class. We assume that this base class has finite Vapnik-Chervonenkis dimension, and we constrain the size of the class by restricting the $\ell_{1}$ norm of the linear parameters. If $\mathcal{G}$ is the VC-class, we write $\mathcal{F}=B \operatorname{absconv}(\mathcal{G})$, for some constant $B$, where

$$
B \operatorname{absconv}(\mathcal{G})=\left\{\sum_{i=1}^{m} \alpha_{i} g_{i}: m \in \mathbb{N}, \alpha_{i} \in \mathbb{R}, g_{i} \in \mathcal{G},\|\alpha\|_{1}=B\right\}
$$




\begin{tabular}{lccc} 
& $\phi(\alpha)$ & $L_{B}$ & $\delta(\epsilon)$ \\
\hline exponential & $e^{-\alpha}$ & $e^{B}$ & $e^{-B} \epsilon^{2} / 8$ \\
logistic & $\ln \left(1+e^{-2 \alpha}\right)$ & 2 & $e^{-2 B} \epsilon^{2} / 4$ \\
quadratic & $(1-\alpha)^{2}$ & $2(B+1)$ & $\epsilon^{2} / 4$ \\
truncated quadratic & $(\max \{0,1-\alpha\})^{2}$ & $2(B+1)$ & $\epsilon^{2} / 4$ \\
\hline
\end{tabular}

Table 1: Four convex loss functions defined on $\mathbb{R}$. On the interval $[-B, B]$, each has the indicated Lipschitz constant $L_{B}$ and modulus of convexity $\delta(\epsilon)$ with respect to $d_{\phi}$. All have a quadratic modulus of convexity.

Theorem 17. Let $\phi: \mathbb{R} \rightarrow \mathbb{R}$ be a convex loss function. Suppose that, on the interval $[-B, B], \phi$ is Lipschitz with constant $L_{B}$ and has modulus of convexity $\delta(\epsilon)=a_{B} \epsilon^{2}$ (both with respect to the pseudometric d).

For any probability distribution $P$ on $\mathcal{X} \times \mathcal{Y}$ that has noise exponent $\alpha$, there is a constant $c^{\prime}$ for which the following is true. For i.i.d. data $\left(X_{1}, Y_{1}\right), \ldots,\left(X_{n}, Y_{n}\right)$, let $\hat{f} \in \mathcal{F}$ be the minimizer of the empirical $\phi$-risk, $R_{\phi}(f)=\hat{\mathbf{E}} \phi(Y f(X))$. Suppose that $\mathcal{F}=B \operatorname{absconv}(\mathcal{G})$, where $\mathcal{G} \subseteq\{ \pm 1\}^{\mathcal{X}}$ has $d_{V C}(\mathcal{G})=d$, and

$$
\epsilon^{*} \geq B L_{B} \max \left\{\left(\frac{L_{B} a_{B}}{B}\right)^{1 /(d+1)}, 1\right\} n^{-(d+2) /(2 d+2)}
$$

Then with probability at least $1-e^{-x}$,

$$
R(\hat{f}) \leq R^{*}+c^{\prime}\left(\epsilon^{*}+\frac{L_{B}\left(L_{B} / a_{B}+B\right) x}{n}+\inf _{f \in \mathcal{F}} R_{\phi}(f)-R_{\phi}^{*}\right) .
$$

Proof. It is clear that $\mathcal{F}$ is convex and satisfies the conditions of Theorem 12. That theorem implies that, with probability at least $1-e^{-x}$,

$$
R(\hat{f}) \leq R^{*}+c^{\prime}\left(\epsilon+\inf _{f \in \mathcal{F}} R_{\phi}(f)-R_{\phi}^{*}\right)
$$

provided that

$$
\epsilon \geq K \max \left\{\epsilon^{*}, \frac{L_{B}^{2} x}{2 a_{B} n}, \frac{B L_{B} x}{n}\right\}
$$

where $\epsilon^{*} \geq \xi_{g_{\mathcal{F}}}\left(\epsilon^{*}\right)$. It remains to prove suitable upper bounds for $\epsilon^{*}$.

By a classical symmetrization inequality (see, for example, Van der Vaart and Wellner, 1996), we can upper bound $\xi_{g_{\mathcal{F}}}$ in terms of local Rademacher averages:

$$
\begin{aligned}
\xi_{g_{\mathcal{F}}}(\epsilon) & =\mathbf{E} \sup \left\{\mathbf{E} g_{f}-\hat{\mathbf{E}} g_{f}: f \in \mathcal{F}, \mathbf{E} g_{f}=\epsilon\right\} \\
& \leq 2 \mathbf{E} \sup \left\{\frac{1}{n} \sum_{i=1}^{n} \epsilon_{i} g_{f}\left(X_{i}, Y_{i}\right): f \in \mathcal{F}, \mathbf{E} g_{f}=\epsilon\right\}
\end{aligned}
$$


where the expectations are over the sample $\left(X_{1}, Y_{1}\right) \ldots,\left(X_{n}, Y_{n}\right)$ and the independent uniform (Rademacher) random variables $\epsilon_{i} \in\{ \pm 1\}$. The Ledoux and Talagrand (1991) contraction inequality and Lemma 15 imply

$$
\begin{aligned}
\xi_{g_{\mathcal{F}}}(\epsilon) & \leq 4 L \mathbf{E} \sup \left\{\frac{1}{n} \sum_{i=1}^{n} \epsilon_{i} d_{\phi}\left(Y_{i} f\left(X_{i}\right), Y_{i} f^{*}\left(X_{i}\right)\right): f \in \mathcal{F}, \mathbf{E} g_{f}=\epsilon\right\} \\
& \leq 4 L \mathbf{E} \sup \left\{\frac{1}{n} \sum_{i=1}^{n} \epsilon_{i} d_{\phi}\left(Y_{i} f\left(X_{i}\right), Y_{i} f^{*}\left(X_{i}\right)\right): f \in \mathcal{F}, \tilde{d}_{\phi}\left(f, f^{*}\right)^{2} \leq 2 a_{B} \epsilon\right\} \\
& =4 L \mathbf{E} \sup \left\{\frac{1}{n} \sum_{i=1}^{n} \epsilon_{i} f\left(X_{i}, Y_{i}\right): f \in \mathcal{F}_{\phi}, \mathbf{E} f^{2} \leq 2 a_{B} \epsilon\right\},
\end{aligned}
$$

where

$$
\mathcal{F}_{\phi}=\left\{(x, y) \mapsto d_{\phi}\left(y f(x), y f^{*}(x)\right): f \in \mathcal{F}\right\} .
$$

One approach to approximating these local Rademacher averages is through information about the rate of growth of covering numbers of the class. For some subset $A$ of a pseudometric space $(S, d)$, let $\mathcal{N}(\epsilon, A, d)$ denote the cardinality of the smallest $\epsilon$-cover of $A$, that is, the smallest set $\hat{A} \subset S$ for which every $a \in A$ has some $\hat{a} \in \hat{A}$ with $d(a, \hat{a}) \leq \epsilon$. Using Dudley's entropy integral (Dudley, 1999), Mendelson (2002) has shown the following result: Suppose that $\mathcal{F}$ is a set of $[-1,1]$-valued functions on $\mathcal{X}$, and there is a $\gamma>0$ and $0<p<2$ for which

$$
\sup _{P} \mathcal{N}\left(\epsilon, \mathcal{F}, L_{2}(P)\right) \leq \gamma \epsilon^{-p}
$$

where the supremum is over all probability distributions $P$ on $\mathcal{X}$. Then for some constant $C_{\gamma, p}$ (that depends only on $\gamma$ and $p$ ),

$$
\frac{1}{n} \mathbf{E} \sup \left\{\sum_{i=1}^{n} \epsilon_{i} f\left(X_{i}\right): f \in \mathcal{F}, \mathbf{E} f^{2} \leq \epsilon\right\} \leq C_{\gamma, p} \max \left\{n^{-2 /(2+p)}, n^{-1 / 2} \epsilon^{(2-p) / 4}\right\} .
$$

Since $d_{\phi}(a, b) \leq|a-b|$, any $\epsilon$-cover of $\left\{f-f^{*}: f \in \mathcal{F}\right\}$ is an $\epsilon$-cover of $\mathcal{F}_{\phi}$, so $\mathcal{N}\left(\epsilon, \mathcal{F}_{\phi}, L_{2}(P)\right) \leq$ $\mathcal{N}\left(\epsilon, \mathcal{F}, L_{2}(P)\right)$.

Now, for the class $\operatorname{absconv}(\mathcal{G})$ with $d_{V C}(\mathcal{G})=d$, we have

$$
\sup _{P} \mathcal{N}\left(\epsilon, \operatorname{absconv}(\mathcal{G}), L_{2}(P)\right) \leq C d \epsilon^{-2 d /(d+2)} ;
$$

(see, for example, Van der Vaart and Wellner, 1996). Applying Mendelson's result shows that

$$
\begin{aligned}
& \frac{1}{n} \mathbf{E} \sup \left\{\sum_{i=1}^{n} \epsilon_{i} f\left(X_{i}\right): f \in B \operatorname{absconv}(\mathcal{G}), \mathbf{E} f^{2} \leq \epsilon\right\} \\
& \leq C_{d} \max \left\{B n^{-(d+2) /(2 d+2)}, B^{d /(d+2)} n^{-1 / 2} \epsilon^{1 /(d+2)}\right\} .
\end{aligned}
$$

Solving for $\epsilon^{*} \geq \xi_{g_{\mathcal{F}}}\left(\epsilon^{*}\right)$ shows that it suffices to choose

$$
\epsilon^{*}=C_{d}^{\prime} B L_{B} \max \left\{\left(\frac{L_{B} a_{B}}{B}\right)^{1 /(d+1)}, 1\right\} n^{-(d+2) /(2 d+2)},
$$

for some constant $C_{d}^{\prime}$ that depends only on $d$. 


\section{Conclusions}

We have focused on the relationship between properties of a nonnegative margin-based loss function $\phi$ and the statistical performance of the classifier which, based on an iid training set, minimizes empirical $\phi$-risk over a class of functions. We first derived a universal upper bound on the population misclassification risk of any thresholded measurable classifier in terms of its corresponding population $\phi$-risk. The bound is governed by the $\psi$-transform, a convexified variational transform of $\phi$. It is the tightest possible upper bound uniform over all probability distributions and measurable functions in this setting.

Using this upper bound, we characterized the class of loss functions which guarantee that every $\phi$-risk consistent classifier sequence is also Bayes-risk consistent, under any population distribution. Here $\phi$-risk consistency denotes sequential convergence of population $\phi$-risks to the smallest possible $\phi$-risk of any measurable classifier. The characteristic property of such a $\phi$, which we term classification-calibration, is a kind of pointwise Fisher consistency for the conditional $\phi$-risk at each $x \in \mathcal{X}$. The necessity of classification-calibration is apparent; the sufficiency underscores its fundamental importance in elaborating the statistical behavior of large-margin classifiers.

For the widespread special case of convex $\phi$, we demonstrated that classification-calibration is equivalent to the existence and strict negativity of the first derivative of $\phi$ at 0 , a condition readily verifiable in most practical examples. In addition, the convexification step in the $\psi$-transform is vacuous for convex $\phi$, which simplifies the derivation of closed forms.

Under the noise-limiting assumption of Tsybakov (2001), we sharpened our original upper bound and studied the Bayes-risk consistency of $\hat{f}$, the minimizer of empirical $\phi$-risk over a convex, bounded class of functions $\mathcal{F}$ which is not too complex. We found that, for convex $\phi$ satisfying a certain uniform strict convexity condition, empirical $\phi$-risk minimization yields convergence of misclassification risk to that of the best-performing classifier in $\mathcal{F}$, as the sample size grows. Furthermore, the rate of convergence can be strictly faster than the classical $n^{-1 / 2}$, depending on the strictness of convexity of $\phi$ and the complexity of $\mathcal{F}$.

Two important issues that we have not treated are the approximation error for population $\phi$-risk relative to $\mathcal{F}$, and algorithmic considerations in the minimization of empirical $\phi$-risk. In the setting of scaled convex hulls of a base class, some approximation results are given by Breiman (2000), Mannor et al. (2002) and Lugosi and Vayatis (2003). Regarding the numerical optimization to

determine $\hat{f}$, Zhang and $\mathrm{Yu}$ (2003) give novel bounds on the convergence rate for generic forward stagewise additive modeling (see also Zhang, 2002). These authors focus on optimization of a convex risk functional over the entire linear hull of a base class, with regularization enforced by an early stopping rule.

\section{Acknowledgments}

We would like to thank Gilles Blanchard, Olivier Bousquet, Pascal Massart, Ron Meir, Shahar Mendelson, Martin Wainwright and Bin Yu for helpful discussions.

\section{A Loss, risk, and distance}

We could construe $R_{\phi}$ as the risk under a loss function $\ell_{\phi}: \mathbb{R} \times\{ \pm 1\} \rightarrow[0, \infty)$ defined by $\ell_{\phi}(\hat{y}, y)=$ $\phi(\hat{y} y)$. The following result establishes that loss functions of this form are fundamentally unlike 
distance metrics.

Lemma 18. Suppose $\ell_{\phi}: \mathbb{R}^{2} \rightarrow[0, \infty)$ has the form $\ell_{\phi}(x, y)=\phi(x y)$ for some $\phi: \mathbb{R} \rightarrow[0, \infty)$. Then

1. $\ell_{\phi}$ is not a distance metric on $\mathbb{R}$,

2. $\ell_{\phi}$ is a pseudometric on $\mathbb{R}$ iff $\phi \equiv 0$, in which case $\ell_{\phi}$ assigns distance zero to every pair of reals.

Proof. By hypothesis, $\ell_{\phi}$ is nonnegative and symmetric. Another requirement of a distance metric is definiteness: for all $x, y \in \mathbb{R}$,

$$
x=y \Longleftrightarrow \ell_{\phi}(x, y)=0 .
$$

But we may write any $z \in(0, \infty)$ in two different ways, as $\sqrt{z} \sqrt{z}$ and, for example, $(2 z)((1 / 2) z)$. To satisfy (14) requires $\phi(z)=0$ in the former case and $\phi(z)>0$ in the latter, an impossibility. This proves 1 .

To prove 2 , recall that a pseudometric relaxes (14) to the requirement

$$
x=y \Longrightarrow \ell_{\phi}(x, y)=0 \text {. }
$$

Since each $z \geq 0$ has the form $x y$ for $x=y=\sqrt{z}$, (15) amounts to the necessary condition that $\phi \equiv 0$ on $[0, \infty)$. The final requirement on $\ell_{\phi}$ is the triangle inequality, which in terms of $\phi$ becomes

$$
\phi(x z) \leq \phi(x y)+\phi(y z), \quad \text { for all } x, y, z \in \mathbb{R} .
$$

Since $\phi$ must vanish on $[0, \infty)$, taking $y=0$ in (16) shows that only the zero function can (and does) satisfy the constraint.

\section{References}

Arora, S., Babai, L., Stern, J., and Sweedyk, Z. (1997). The hardness of approximate optima in lattices, codes, and systems of linear equations. Journal of Computer and System Sciences, $54: 317-331$.

Bartlett, P. L. (1998). The sample complexity of pattern classification with neural networks: the size of the weights is more important than the size of the network. IEEE Transactions on Information Theory, 44(2):525-536.

Bartlett, P. L., Bousquet, O., and Mendelson, S. (2003). Local Rademacher complexities. Technical report, University of California at Berkeley.

Boser, B. E., Guyon, I. M., and Vapnik, V. N. (1992). A training algorithm for optimal margin classifiers. In Proceedings of the 5th Annual Workshop on Computational Learning Theory, pages 144-152, New York. ACM Press.

Bousquet, O. (2002). A Bennett concentration inequality and its application to suprema of empirical processes. Comptes Rendus de l'Académie des Sciences, Série I, 334:495-500. 
Boyd, S. and Vandenberghe, L. (2003). Convex Optimization. Stanford University, Department of Electrical Engineering.

Breiman, L. (2000). Some infinity theory for predictor ensembles. Technical Report 577, Department of Statistics, University of California, Berkeley.

Brown, L. D. (1986). Fundamentals of Statistical Exponential Families. Institute of Mathematical Statistics, Hayward, CA.

Collins, M., Schapire, R. E., and Singer, Y. (2002). Logistic regression, Adaboost and Bregman distances. Machine Learning, 48:253-285.

Cortes, C. and Vapnik, V. (1995). Support-vector networks. Machine Learning, 20:273-297.

Cristianini, N. and Shawe-Taylor, J. (2000). An Introduction to Support Vector Methods. Cambridge University Press, Cambridge.

Devroye, L., Györfi, L., and Lugosi, G. (1996). A Probabilistic Theory of Pattern Recognition. Springer, New York.

Dudley, R. M. (1999). Uniform Central Limit Theorems. Cambridge University Press, Cambridge.

Freund, Y. and Schapire, R. E. (1997). A decision-theoretic generalization of on-line learning and an application to boosting. Journal of Computer and System Sciences, 55(1):119-139.

Friedman, J., Hastie, T., and Tibshirani, R. (2000). Additive logistic regression: A statistical view of boosting. Annals of Statistics, 28:337-374.

Jiang, W. (2003). Process consistency for Adaboost. Annals of Statistics, in press.

Jordan, M. I., Ghahramani, Z., Jaakkola, T. S., and Saul, L. K. (1999). Introduction to variational methods for graphical models. Machine Learning, 37:183-233.

Klein, T. (2002). Une inégalité de concentration à gauche pour les processus empiriques. [A left concentration inequality for empirical processes]. Comptes Rendus de l'Académie des Sciences, Série I, 334(6):501-504.

Koltchinskii, V. and Panchenko, D. (2002). Empirical margin distributions and bounding the generalization error of combined classifiers. Annals of Statistics, 30(1):1-50.

Lebanon, G. and Lafferty, J. (2002). Boosting and maximum likelihood for exponential models. In Advances in Neural Information Processing Systems 14, pages 447-454.

Ledoux, M. (2001). The Concentration of Measure Phenomenon. American Mathematical Society, Providence, RI.

Ledoux, M. and Talagrand, M. (1991). Probability in Banach Spaces: Isoperimetry and Processes. Springer, New York.

Lee, W. S., Bartlett, P. L., and Williamson, R. C. (1996). Efficient agnostic learning of neural networks with bounded fan-in. IEEE Transactions on Information Theory, 42(6):2118-2132. 
Lin, Y. (2001). A note on margin-based loss functions in classification. Technical Report 1044r, Department of Statistics, University of Wisconsin.

Lugosi, G. and Vayatis, N. (2003). On the Bayes risk consistency of regularized boosting methods. Annals of Statistics, in press.

Mannor, S. and Meir, R. (2001). Geometric bounds for generalization in boosting. In Proceedings of the Fourteenth Annual Conference on Computational Learning Theory, pages 461-472.

Mannor, S., Meir, R., and Zhang, T. (2002). The consistency of greedy algorithms for classification. In Proceedings of the Annual Conference on Computational Learning Theory, pages 319-333.

Massart, P. (2000a). About the constants in Talagrand's concentration inequality for empirical processes. Annals of Probability, 28(2):863-884.

Massart, P. (2000b). Some applications of concentration inequalities to statistics. Annales de la Faculté des Sciences de Toulouse, IX:245-303.

Mendelson, S. (2002). Improving the sample complexity using global data. IEEE Transactions on Information Theory, 48(7):1977-1991.

Nesterov, Y. and Nemirovskii, A. (1994). Interior-Point Polynomial Algorithms in Convex Programming. SIAM Publications, Philadelphia.

Rio, E. (2001). Inégalités de concentration pour les processus empiriques de classes de parties [Concentration inequalities for set-indexed empirical processes]. Probability Theory and Related Fields, 119(2):163-175.

Rockafellar, R. T. (1997). Convex Analysis. Princeton University Press, Princeton, NJ.

Schapire, R. E., Freund, Y., Bartlett, P., and Lee, W. S. (1998). Boosting the margin: A new explanation for the effectiveness of voting methods. The Annals of Statistics, 26(5):1651-1686.

Schölkopf, B. and Smola, A. (2002). Learning with Kernels. MIT Press, Cambridge, MA.

Shawe-Taylor, J., Bartlett, P. L., Williamson, R. C., and Anthony, M. (1998). Structural risk minimization over data-dependent hierarchies. IEEE Transactions on Information Theory, 44(5):1926-1940.

Steinwart, I. (2002). Consistency of support vector machines and other regularized classifiers. Technical Report 02-03, University of Jena, Department of Mathematics and Computer Science.

Talagrand, M. (1994). Sharper bounds for Gaussian and empirical processes. Annals of Probability, $22(1): 28-76$.

Tsybakov, A. (2001). Optimal aggregation of classifiers in statistical learning. Technical Report PMA-682, Université Paris VI.

Van der Vaart, A. W. and Wellner, J. A. (1996). Weak Convergence and Empirical Processes. Springer-Verlag, New York. 
Zhang, T. (2002). Sequential greedy approximation for certain convex optimization problems. Technical Report RC22309, IBM T. J. Watson Research Center, Yorktown Heights.

Zhang, T. (2003). Statistical behavior and consistency of classification methods based on convex risk minimization. Annals of Statistics, in press.

Zhang, T. and Yu, B. (2003). Boosting with early stopping: Convergence and consistency. Technical Report 635, Department of Statistics, University of California, Berkeley. 